\title{
Review
}

\section{Microbiota-gut-brain-axis and Autism Spectrum disorders}

\author{
Piranavie Srikantha, M. Hasan Mohajeri* \\ Department of medicine, University of Zurich, Winterthurerstrasse 190, 8057 Zürich, Switzerland. \\ piranavie.srikantha@uzh.ch, mhasan.mohajeri@uzh.ch \\ * Correspondence: mhasan.mohajeri@uzh.ch; Tel.: +41-79-938-1203
}

\begin{abstract}
New research points to a possible link between Autism Spectrum Disorder and the gut microbiota as many autistic children have co-occurring gastrointestinal problems. This review focuses on specific alterations of gut microbiota mostly observed in autistic patients. Particularly, the mechanisms through which such alterations may trigger the production of the bacterial metabolites or leaky gut in autistic people are described. Various altered metabolite levels were observed in autistic children, many of those were of bacterial origin such as short chain fatty acids (SCFAs), indoles and lipopolysaccharides. A less integrative gut-blood-barrier is abundant in autistic individuals. This explains the leakage of bacterial metabolites into the patients triggering new body responses or altered metabolism. Some other co-occurring symptoms such as mitochondrial dysfunction, oxidative stress in the cells, altered tight junctions in the blood brain barrier and structural changes in cortex, hippocampus, amygdala and cerebellum were detected. Moreover, this paper suggests that autism is associated with an unbalanced gut microbiota (dysbiosis). Although the cause-effect relationship between autism and gut microbiota is not yet well established, consumption of specific probiotics may represent a powerful tool to re-establish gut homeostasis and promote gut health. Diagnostic and therapeutic value of new biomarkers leading to the perturbation in the phenylalanine metabolism will be discussed.
\end{abstract}

Keywords: Autism, ASD, microbiota, gut-brain-axis, microbiota-gut-brain-axis, therapy

\section{Introduction}

The microbiota-gut-brain-axis describes the bidirectional physiological connection to exchange information between the microbiota, the gut and the brain [1]. The term microbiota describes the entirety of all bacterial, archaeal and fungal microorganisms living on our skin, in the mouth, in the respiratory, gastrointestinal (GI) and vaginal tracts [2]. The microbial composition varies through age, diet, diseases, geography or travel [2,3]. The highest diversity of microbiota is found in the large intestine [4]. The GI tract, the largest surface in the body, meets trillions of microorganisms separated by the gut barrier [5]. The gut barrier is made out of the commensal gut microbiota, a mucus layer and epithelial cells connected through tight junctions [6]. Gut microbiota interacts with the human metabolism by educating the immune system, protecting against pathogenic microbes, metabolising non-digestible carbohydrates, producing essential vitamins and antimicrobial substances [7-10]. In addition, commensal bacteria reinforce the gut barrier by increasing mucine production [11] and even stimulating intestinal angiogenesis [12]. GI symptoms are four times more prevalent in children with autism compared to the normal population [13] affecting men and boys more than women and girls [14]. It is known that many autistic children take relatively high doses of oral antibiotics in early years of life, which could be the factor disturbing the gut microbiota [15]. Dysbiosis is an altered microbial composition favouring pathogenic microbes over beneficial ones in the gut. Knowing that dysbiosis in young ages affects health-status during life, it is important to study the life-long impacts of compromised gastrointestinal functions during childhood. Dysbiosis is often observed in gut-related diseases and conditions such as inflammatory bowel disease, irritable bowel syndrome, diabetes and 
obesity, but also in unconventional diseases such as acne, stress, cardiovascular disease, Alzheimer's disease, Multiple Sclerosis, Parkinson's and Autism Spectrum disorders [16-20].

The term Autism Spectrum disorder (ASD) does not only include autism, but also Asperger's Syndrome and Pervasive Developmental Disorder Not Otherwise Specified [7]. Asperger's Syndrome manifests next to social withdrawal with superior cognitive skills [21]. Regressive autism or late onset autism describes a subgroup of patients with initially normal development but with a gradual loss of acquired skills in communication or social interaction [7]. Autistic behaviour is characterized by poor communication skills, social withdrawal, repetitive or restrictive pattern in behaviour, interests and activities, picky eating habits, [22-24] increased aggression [25] and anxiety [26]. Abnormal eating habits may lead to deficiencies in vitamins, minerals and fatty acids in autistic individuals [23]. The prevalence of autism spectrum disorder was one in fifty-nine children in the United States in 2014 [27]. Most cases of autism are idiopathic. The phenotypes of ASD are heterogeneous, suggesting that many factors play into the aetiology [28]. Both genetic and environmental factors are implicated in autism [15]. Environmental factors, among others, regulate gene expression through epigenetic modulation [29].

The frequently reported comorbidity of GI problems in autistic children such as constipation, diarrhoea, bloating, abdominal pain, reflux, vomiting, gaseousness, foul smelling stools and food allergies $[1,4,8,23,30-32]$, motivated the central question of this review: Is there a connection between the gut microbiota and the central nervous system (CNS) leading to the pathology of autism? This is an important question considering that only approximately one third of all autistic cases could be linked to genetic causes [33]. Furthermore, no effective treatment is available for Autism Spectrum Disorder to date. Therefore, understanding and modulating the microbiota-gut-brain-axis may be an effective and promising way for treating and ameliorating disease symptoms [2]. The objective of this review is to summarize all human studies and reviews on the topic connecting gut microbiota with autism. We highlight here the importance of new biomarkers showing perturbation in the phenylalanine metabolism. We provide the evidence that 3-(3-hydroxyphenyl)-3-hydroxypropionic acid, 3-hydroxyphenylacetic acid and 3-hydroxyhippuric acid are significantly elevated in autistic children [34] and are related to the abundance of Clostridia species which worsens autistic behaviour [34]. These data might be helping to start treating autistic individuals prior to the onset of GI problems.

\section{Material and Methods}

For this systematic review PubMed databank was searched on $1^{\text {st }}$ March 2018 using the search terms „Microbiome OR gut-brain-axis AND autism“. Only human studies and full text publications were considered. Non-English papers were excluded and a total of 126 papers were examined for this paper. All original works are cited in this paper. Some reviews were not cited due to repetitions in content among the reviews. 


\section{Comorbidities in Autism}

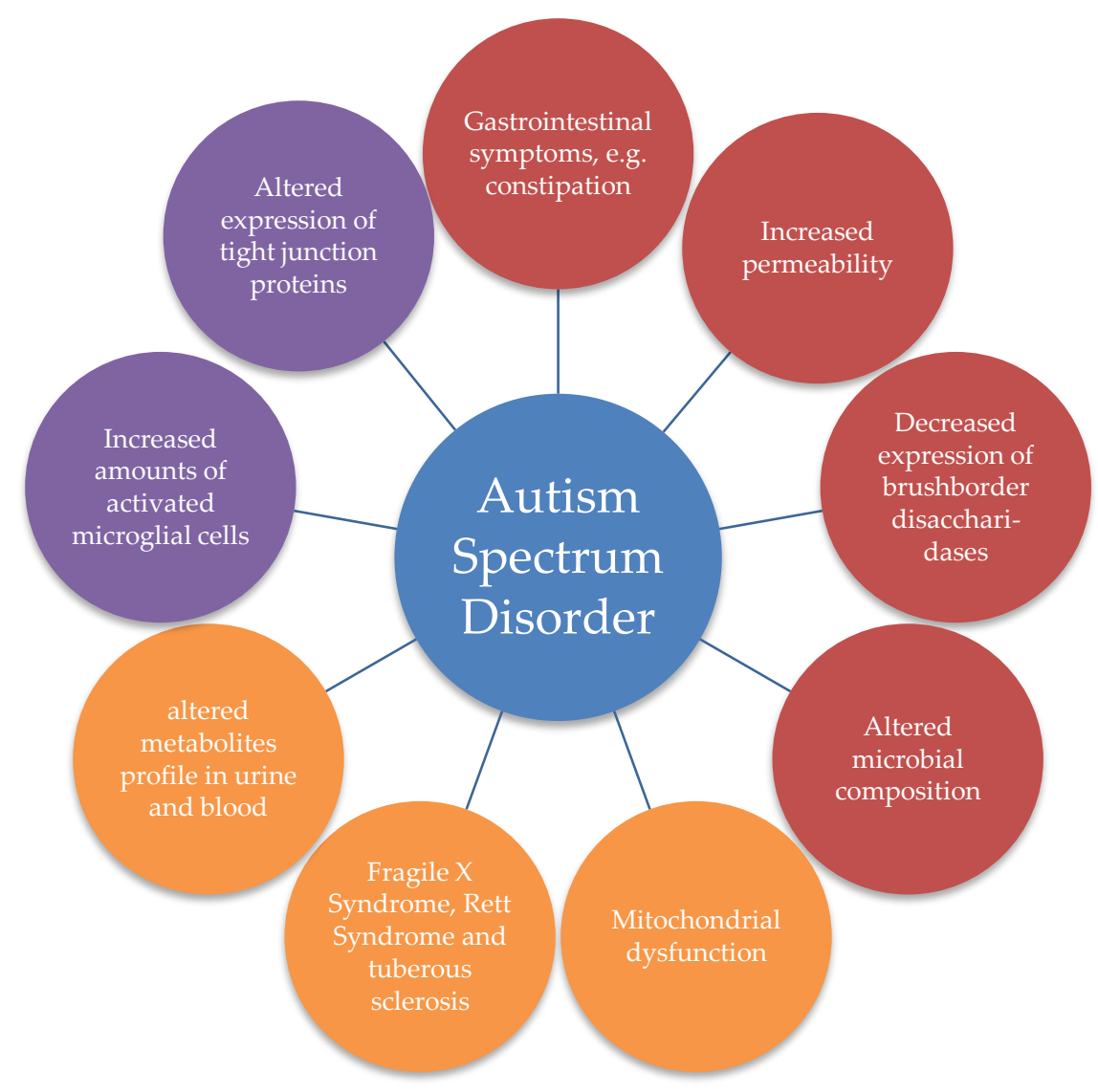

Figure 1. Co-occurring pathologies found in Autism Spectrum disorder (ASD). Red shows gut-related comorbidities found in ASD, purple shows brain-related comorbidities and orange other comorbidities. Chapter 3 gives more information to the mentioned comorbidities.

\subsection{Gastrointestinal symptoms}

GI problems are often observed in autistic individuals (figure 1) [1,4,8,23,30-32]. In a small sample size severity and number of GI symptoms were found to be significantly higher in autistic children and their non-autistic siblings compared to healthy controls [35]. Accordingly, a different bigger study comparing 230 pre-schoolers discovered similar results, that ASD patients were suffering significantly more often from GI problems than healthy controls. ASD patients with GI symptoms were found to have more anxiety problems and more other somatic complaints in addition to less social interaction in comparison to ASD patients without GI problems [30]. Constipation was found to be the most common GI symptom observed in autistic children. The same authors also found a connection between rigid-compulsive behaviour and the occurrence of constipation [13]. Additionally, GI problems in autistic children lead to more tantrums, aggressive behaviour and sleep disturbances, further worsening the behaviour compared to autistic individuals without GI symptoms [8]. One review suggested that altered behaviour such as aggression, self-injury or sleep disorder observed in autistic children might be an expression of abdominal discomfort [36].

Autistic children show an altered expression and metabolism of disaccharides and hexose transporters in their gut epithelium (figure 1) [15,37], such as low activity of lactase in the small intestine [15]. The disaccharidases in the brush border of the ileum, lactase, maltase glucoamylase and sucrose isomaltase, were found to have lower mRNA levels and hence a decreased gene expression [38,39]. The sodium-dependent glucose cotransporter (SGLT1) and the glucose transporter 2 (GLUT2) transport glucose, galactose and fructose actively across the luminal and basolateral membranes of enterocytes. Once more, autistic children had significantly decreased mRNA levels of both hexose transporters in the ileum [39]. As a consequence of these decreased gene 
expressions, more mono- and disaccharides enter the large intestine. Thus, bacteria fermenting these sugars profit and outcompete bacteria degrading polysaccharides leading to an altered microbial composition in the GI-tract. Higher amounts of sugars in the large intestine could lead to osmotic diarrhoea or these sugars serve as substrates for the production of gases [39]. Diarrhoea and bloating are both GI symptoms observed in ASD patients. GI symptoms in ASD patients are known to correlate with autistic behaviour [8].

\subsection{Increased gut permeability}

An increased intestinal permeability was observed in ASD patients (figure 1) measuring lactulose in blood after oral administration [8]. Autistic children as well as their non-autistic siblings were found to have increased gut permeability compared to controls [40]. Increased gut permeability is probably a result of decreased expression of barrier forming proteins and elevated expression of pore forming proteins of tight junctions. These altered expressions were found by analysing mucosal samples from the small intestine of autistic children compared to controls [41]. Changed permeability could lead back to lower counts of Lactobacillus strains in autistic patients, as they are associated with the maintenance of tight junction in the epithelial barrier of the intestines [8]. Increased intestinal permeability was also found in some close non-autistic relatives of autistic individuals. This suggests that intestinal integrity is not a consequence of autism as the relatives were non-autistic. Rather, increased gut permeability should be viewed as a cause, which could in combination with other environmental factors, at least partially, lead to the pathology of autism [42].

\subsection{Alterations in the brain}

Analysis of post-mortem brains of autistic patients revealed altered expression of some claudines (CLDN) in the blood-brain-barrier (BBB) of the cortex and cerebellum. Significant changes were observed in the expression of the genes of CLDN-5 and CLDN-12. These two major constituents of tight junctions were overexpressed in the cortex and cerebellum of autistic children compared to controls (figure 1). CLDN-5 is an important protein in tight junctions for cell adhesion of endothelial cells in the brain. However, the protein expression of CLDN-12 was significantly reduced in the cortex of autistic children even though the gene was overexpressed [41]. The over-expressions of the genes are suggestive of either a higher integrity of the BBB in autistic children or a compensatory mechanism to uphold the integrity of the BBB. The second explanation appeared more plausible, as an increased destruction of the protein CLDN-12 could lead to an over-expression of the CLDN-12 gene and other tight junction components as CLDN-5. These results suggest a lower integrity of the BBB leading to a compensatory overexpression of tight junction components.

Increased activation of microglial cells and other structural changes have also been observed post-mortem in the brains of autistic individuals (figure 1) [28,43]. The increase in activation of microglial cells suggests that autism is a pathology caused or at least accompanied by immune activation in the brain. Inflammation in the brain could subsequently lead to malfunctioning synapses [28]. During inflammation the brain releases arginine vasopressin. Arginine vasopressin is a metabolite, which is known to act on social behaviour and is a considered biomarker for autism [28]. A structural change was the increased weight of ASD brains possibly due to the presence of a larger number of neurons in the prefrontal cortex [43]. Moreover, Purkinje cells occurred in decreased numbers in the cerebellum of ASD patients [27,44]. Purkinje cells produce GABA, which makes them vulnerable to the tetanus neurotoxin produced by Clostridia tetani [27]. Elevated amounts of Clostridia species could account for decreased Purkinje cells in the cerebellum.

\subsection{Mitochondrial Dysfunction}

Mitochondrial dysfunction was detected in ten ASD children (figure 1) after comparing intestinal mucosa samples of rectum und caecum with that of ten Crohn's disease children and ten healthy children. The children with Crohn's disease represented non-autistic children with GI symptoms, whereas healthy children had no GI problems [45]. ASD children displayed lower activity of the electron transport chain (ETC) enzyme complex IV and citrate synthase in mucosal samples 
taken from rectum and caecum. In addition, they also showed significantly higher quantity of ETC complex I in the mucosa of the caecum. The quantities of the proteins from ETC enzyme complexes III, IV and V were only significantly increased in the caecum [45]. Mitochondrial dysfunctions disrupt the function of enterocytes and therefore cause gut-dysmotility and higher sensitivity to oxidative stress. Increased oxidative stress in the cell leads to damage of proteins and lipids and consequently causes diminished function of enterocytes. The occurrence of these changes only in the caecum and not also in rectum is intriguing. Especially so since the rectum acts a transition passage, whereas the caecum is meant for a longer stay. This is suggestive of bacterial metabolites being involved in mitochondrial dysfunction in the caecum. SCFAs produced by Clostridia species could enter the mitochondria and be utilized as substrates for energy production [45]. For example, butyrate is converted into acetyl-CoA, which then is utilized in the citric cycle for NADH production. NADH on the other hand, is a substrate for ETC complex I, which could be the factor elevating its activity. Additionally, gut-dysmotility caused by mitochondrial dysfunction would explain constipation observed in autistic individuals [45]. The occurrence of many GI problems observed in autistic children could partially be explained by gut-dysmotility. Interestingly GI problems similar to that of autistic children have also been noted in children with mitochondrial dysfunction [45].

\section{Factors influencing the Gut Microbiota}

\subsection{Definitions and Facts}

As the term microbiota describes all microorganisms living in and on humans, the term microbiome describes all the genes expressed in all these microorganisms [36]. The two most dominant bacterial phyla in the gut of healthy humans are Firmicutes and Bacteroidetes; other high abundant phyla are Proteobacteria, Verrucomicrobia and Actinobacteria. These five phyla make up $97.2 \%$ of the gut microbial composition in adult individuals $[7,15,46,47]$. The estimation is that around 1000 different species of bacteria live in the GI tract [48]. Higher diversity of gut microbiota can help maintaining a healthy gut flora in preventing pathogenic bacteria from growing [15]. Diet and environmental factors are among the biggest influencers of microbial composition in healthy adults. This can be seen in people from Malawi or Burkina Faso, as they have a higher diversity in the gut microbiota than people living in the USA. The differences could occur due to altering diets, exercise and hygiene [49,50].

\subsection{Developmemt and disruptions of microbial colonisation potentially leading to autism}

Colonisation of the GI tract with microbiota starts prenatally as microorganisms have been detected in the placenta and meconium, which is the first stool of a new-born [4,51-53]. Moreover, some bacterial species of Enterococcus, Streptococcus and Staphylococcus were found in the blood of the umbilical cord. This suggests a transfer of microbiota from the mother to the foetus [54]. Thus, infants are not born with a sterile GI tract. A new-born shows less microbial diversity and a dominance of the phyla Proteobacteria in the gut. With time the diversity and colonisation increase and the dominance in Firmicutes and Bacteroidetes can be observed. The intestinal microbiota of new-borns is very instable and highly dynamic [55]. First colonisers of healthy new-borns are Enterobacteria, Staphylococcus and Streptococcus species, which are facultative anaerobes. These bacteria consume the oxygen in the gut and so create an anaerobic environment. Following these first colonisations strict anaerobes as Bacteroides, Bifidobacterium and Clostridium species can colonise the gut [55]. The composition of the microbiota stabilises around the age of $2-3$ years $[4,56]$. Interestingly the brain of neonates also grows from $36 \%$ to approximately $90 \%$ of its future adult volume until the age of 2 . The formation of new synapses in the brain also peaks between the age of 3 months and 2 years [29]. The critical window for establishment of a healthy microbial composition falls into the same critical time window for brain development, specifically the formation of new synapses [29]. During the development the brain is sensitive to many external and internal environmental factors. Therefore, it is postulated that changes in prenatal maternal stress level, infections or diet can lead to neurodevelopmental disorders [54,57]. 
Vaginally born babies who are breastfed have the healthiest composition of microbiota. Beneficial bacteria as Bifidobacteria are more abundant than pathogenic bacteria as Clostridium difficile or Escherichia coli [12], as human breast milk contains and promotes growth of the bacterium Bifidobacterium longum through oligosaccharides [51]. Vaginally born babies have a microbiota that resembles vaginal microbiota composition of the mother with dominance of Lactobacillus, Prevotella and Snethia species [58]. Neonates born via caesarean section have an altered microbiota composition resembling the microbiota of the mother's skin with dominance of Staphylococcus, Corynebacterium and Propionibacterium species [58,59]. Exclusively breastfed infants display also richness in Bifidobacterium species, which are specialised in digesting oligosaccharides contained in human milk [58]. Preterm born babies have low levels of gastric acidity, as GI functions have not fully matured. Consequently, more pathogenic microbes survive the stomach and can colonise the intestines leading to an accumulation of pathogenic microbiota and less diversity in the GI tract [17]. They display dominance in Proteobacteria and lack in genera as Lactobacillus and Bifidobacterium [56]. Higher risk for developing Autism were observed in children, (i) who were born pre-term with a very low birthweight [51], (ii) who were born by caesarean delivery, (iii) who were not breastfed, (iv) who underwent prolonged hospitalization or (v) who were treated with antibiotics for a long period [23]. A study found that autistic children experienced significantly shorter period of breastfeeding [60]. Preeclampsia during pregnancy also has an impact on neurodevelopment, increasing the risk for autism and cognitive delay in general [61].

Using information from a population-based registry with approximately 2.6 million children, a Swedish study noted that elective caesarean births in comparison to natural vaginal delivery is associated with a significantly $20 \%$ higher risk of the child developing autism [62]. In contrast, no association was found in the sibling control analysis so the authors concluded that there was no link between caesarean birth and autism at all [62]. Another smaller cohort study from the UK with over $18^{\prime} 000$ participants also found no association found between caesarean birth and development of autism [63]. A possible explanation of this discrepancy was unrevealed by Gondalia et al. who analysed microbial composition of 51 autistic children and their 53 non-autistic siblings and found no significant difference in composition [46]. Similarly, a different study including 59 autistic children and 44 non-autistic siblings also found no significant difference in the composition of the gut microbiota [64]. These data suggest that the microbiota of siblings are similar and independent from their autistic phenotype. Therefore, one cannot use siblings as control to represent the whole population [46]. Similar microbial composition of autistic individuals and their siblings can be a result of shared environment and genetic makeup [65].

Antibiotic treatment during the first three years of life can have detrimental effect on normal microbiota establishment with long-term effects [66]. Gut microbiota reacts strongly to antibiotics by decreasing the variety of species. Recovery of the microbiota to the pre-treatment state is incomplete even several months after the cessation of the treatment [2]. Early treatment of infants with antibiotics can therefore disrupt the colonisation of Bifidobacteria [56]. Changes in the composition of the microbiota could lead to a leaky gut or increased passage of metabolites such as lipopolysaccharides or SCFAs, both produced by bacteria, through intestinal barrier $[1,67]$. These metabolites can activate an inflammatory response through binding to Toll-like receptor 4 also affecting the CNS [1].

Another aspect, leading to a modified microbial composition in infants and increased risk of autism, is maternal infection during pregnancy. This was discovered in maternal immune activated mice (MIA). Pregnant mice were injected with polyinosinic:polycytidylic acid (poly(I:C)) to simulate viral infections. The offspring, which are called MIA mice, displayed altered microbial composition, GI barrier defects (or leaky gut) and behaviour similar to autistic features [16]. Elevated levels of cytokines in maternal blood were proposed to be the causing factor as poly(I:C) is also a cytokine [16]. Elevated levels of IL-6 were found in the colon from MIA mice [16]. The cytokine IL-6 is known to alter the expression of tight junction proteins, as it is proinflammatory, thus helping white blood cells to migrate through epithelial barriers [16]. Hence, the increased gut permeability in autistic children could be a consequence of immune activation in the GI tract [68]. IL-6 also plays a role in synaptic plasticity, cognition and neurodevelopment [69]. The microbial composition of MIA mice showed increased levels of Porphyromonadaceae, Prevotellaceae and decreased levels of 
Ruminococcaceae, Erysipelotrichaceae and Alcaligenaceae [16]. Bacteroides, Bifidobacterium and Lactobacillus species create an anti-inflammatory milieu, whereas Clostridium species stimulate a pro-inflammatory milieu [70]. Cytokines produced by microbiota affected the oligodendrocytes. Therefore, an inflammation during pregnancy can result in behavioural changes in the infant, as Autism Spectrum disorder [71]. Women were found to have a higher risk of carrying out autistic children, when they were hospitalised due to an infection during pregnancy. Interestingly, the risk for autism was higher when infection occurred during a critical time window. Viral infections in the first trimester of the pregnancy and bacterial infections in the second trimester were associated with a higher risk of birthing autistic children [28].

\subsection{Connections between the microbiota and the brain}

There are four known ways serving as bidirectional communication pathways between the gut microbiota and the CNS: (i) afferent and efferent neuronal pathways as for example the vagal nerve [71-75], (ii) the immune system through cytokines, (iii) metabolites produced by the bacteria or the neuroendocrine system of the gut acting on the brain and (iv) the HPA-axis, stress hormones acting on gut function [71,76]. The vagal nerve was suggested as a pathway of communication between microbiota and brain after probiotic treatment in mice resulting in behavioural changes. The same result could not be reproduced in vagotomised mice [56,77]. The vagal nerve contains more afferent than efferent fibres, whereby this pathway communicates mostly from the gut to the brain than vice versa [78]. Several hundred million neurons connect the brain with the gut. Bacterial metabolites can act upon these neurons as neuromodulators [79]. The microbiota is in constant contact with the immune system at the luminal surface of the GI tract [55]. Approximately $70 \%$ of all immune cells of the human body lay in the gut-associated lymphoid tissue. These cells sense alteration of microbial composition and metabolites through receptors [48]. The GI immune cells are immune-tolerant to beneficial microbes. Contact to pathogenic microbes on the other hand results in an inflammatory response. Altered microbial composition might lead to an altered milieu of pro- and antiinflammatory cytokines, which may affect the brain [55].

\subsection{Breakthroughs from studies performed in germ-free mice}

Germ-free (GF) mice are used to study the impact of the microbiota on the host, as GF mice have no microbiota. GF mice are produced by removal of the mice pubs after caesarean births and raising them in an isolated sterile environment $[71,80]$ or treating normal mice with broad-spectrum antibiotics [81]. In contrary to GF mice, conventionally raised mice show an established diverse microbiota. Studies of GF mice presented morphological, structural, and functional abnormalities not seen in conventionally raised mice (figure 2) [80,82]. This steered the biological research towards the relationship between the microbiota and brain diseases in the first place. The dominances of the phyla Bacteroidetes and Firmicutes is also observed in the murine microbiota, however, approximately 85 $\%$ of bacterial genera of the mouse are not found in humans [83]. Therefore, the validity of drawing conclusions from GF mice to humans is limited.

Several changes were found to be important in the brain and behaviour of GF mice. GF mice showed higher expression rates of the myelin genes, resulting in thicker myelin sheaths in the prefrontal cortex associated with fear and social behaviour [71]. Brain derived neurotropic factor (BDNF) expression is decreased in GF mice [22]. Increased neurogenesis in the dorsal hippocampus was observed in these mice [22]. Furthermore, immature microglial cells were found numerously in the cortex and white matter of the cerebellum, corpus callosum, hippocampus and olfactory bulb from GF mice [81]. Immature microglial cells showed a decreased response to pathogens [29]. In a mouse model with elevated immature microglial cells, the formation of more immature synapses was observed [28]. An underdeveloped immune response in the brain in combination with a leaky BBB [53] could result in undesirable metabolites or even pathogens entering the CNS. Changes were also observed in the amygdala, which is important for fear-related and social behaviour [22]. A hypertrophy in dendrites and an overall increased volume in the basolateral amygdala were reported in GF mice [22]. Structural modifications or functional alterations in the amygdala are related to 
autism, so the changes observed in the amygdala could be responsible for ASD-like behaviour in GF mice [84]. Bacterial metabolites carried to the CNS by the vagal nerve could reach the amygdala, resulting in these observed changes [42]. GF mice developed decreased anxiety and social behaviour compared to conventionally raised mice [85]. In a social experiment, GF mice and conventionally raised mice were introduced to an unknown mouse and a new object. Conventionally raised mice spent more time with the new mouse, whereas GF mice preferred to spend more time with the new object. This experiment confirmed impaired social behaviour as observed in ASD patients in GF mice [86]. GF mice in general showed less sociable behaviour than controls [17,87]. Interestingly, antibiotic treatment of conventionally raised mice led to behavioural changes resembling ASD symptoms, whereas no change was observed in GF mice after administration of antibiotics [56]. Observed changes in GF mice are summarized in Figure 2.

Introduction of the microbiota in the pre-weaning period could reverse the neuronal and behavioural changes observed in GF mice. Rehabilitation of the microbiota post-weaning on the other hand, could not reverse or ameliorate the changes. This result suggested that re-colonisation of the microbiota must be executed in a certain time window during the development of the pups to influence brain development through microbiota [22,85].

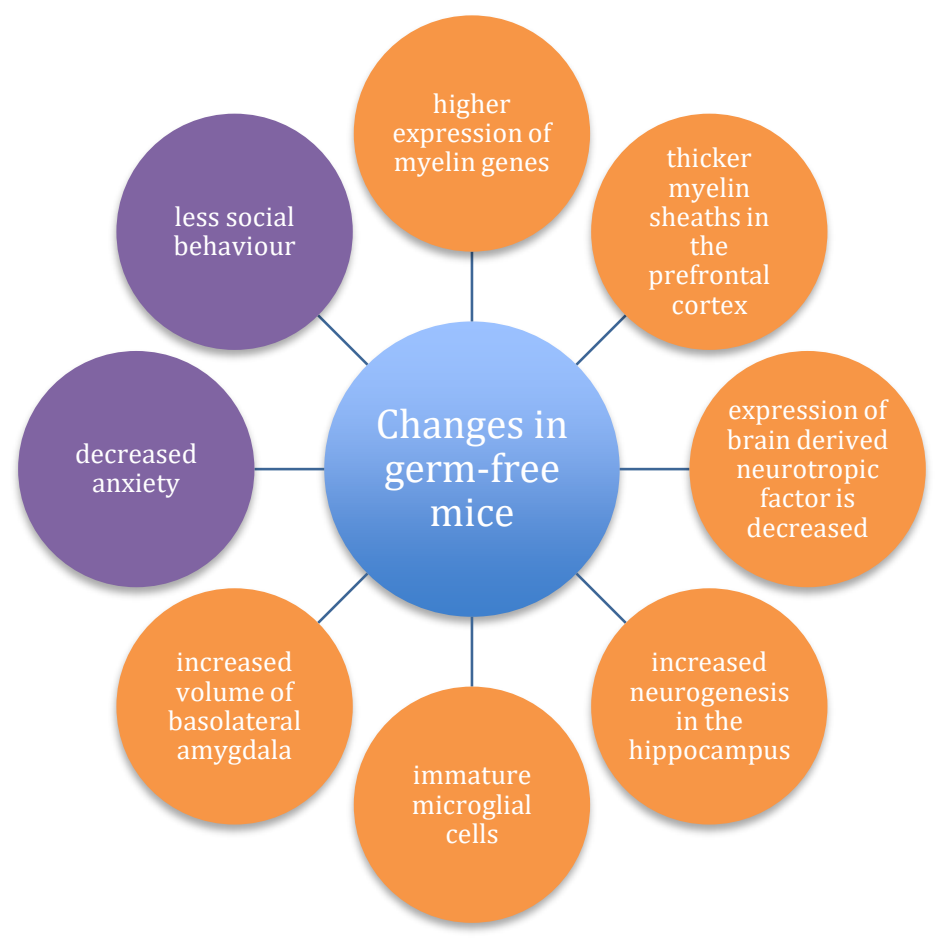

Figure 2. Functional and structural differences in the brain and behaviour of GF compared to normal mice. Orange indicates structural or morphological changes observed in germ free (GF) mice, purple indicates behavioural changes observed in GF mice.

\section{Alterations in microbial composition and metabolic profile in autistic individuals}

\subsection{Changes in microbial composition in autistic children compared to healthy children}

Dysbiosis is the term referring to an altered composition of the microbiota with more pathogenic than beneficial microbes influencing the health of the host [42]. This is the case, for example, after intake of antibiotics at young ages resulting in eradication of protective microbiota. Diarrhoea is also a disrupting factor of the microbiota soothing the path for pathogens [27]. Dysbiosis has been observed in diseases and conditions such as autism (figure 3), allergy, depression, inflammatory bowel disease and obesity [88]. Change in gut microbial composition at young age can affect disease progression later in life, as the microbiota is not yet fully established [27]. 


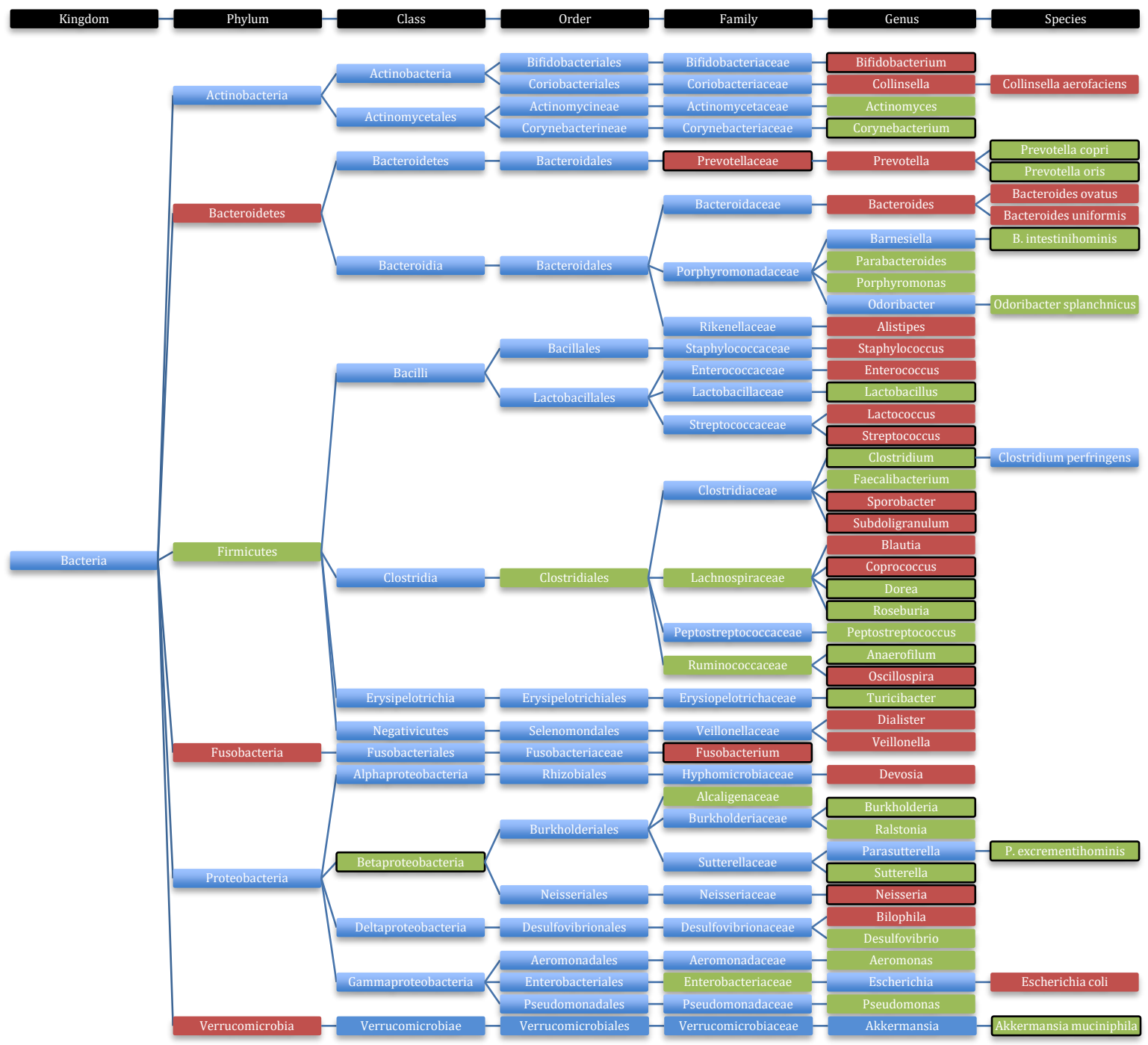

Figure 3. Taxonomic classification of dysbiotic bacteria found in autistic individuals. All bacterial taxa mentioned within this chapter are found in this figure. Green indicates elevated taxa in the gut microbiota of ASD individuals. Red indicates decreased taxa in the gut microbiota ASD individuals. Additionally, black surrounded boxes show significant results in at least one study.

A regularly observed phenomenon in the faeces of autistic children is a significantly decreased ratio between the phyla Bacteroidetes to Firmicutes [35,39,58,89], which pointed at elevated numbers of Firmicutes in contrast to decreased levels of Bacteroidetes. A decreased ratio of these two phyla was also found in inflammatory bowel disease or obesity [89]. Conversely, another study with thirty subjects found that Firmicutes counts were lower and Bacteroidetes were higher represented in autistic children when compared to healthy controls, however, these results were not significant [7]. Kang et al. compared the intestinal flora of twenty autistic children with GI problems to the intestinal flora of twenty neurotypical children. They found that the significant lower bacterial diversity found in autistic children [15,90] correlates with the severity of GI symptoms. No major differences were detected at phylum level, however, this discrepancy could be a result of the parents collecting and freezing the stool samples and not the research team. Neurotypical children had a significantly higher abundance of the genus Coprococcus and class Prevotellaceae compared to autistic faecal samples. Species from the genus Prevotella are commensal gut microbes, specialised in degrading plant polysaccharides and synthesising vitamin B1. Lower abundance of Prevotella species could result in a vitamin B1 deficiency. In addition, Veillonellaceae were found in lower abundance in autistic 
children. Small differences were observed in Sutterella genus, being less abundant in autistic children [15].

Intestinal biopsies from the caecum and the terminal ileum of ASD children showed significantly increased numbers of Sutterella species compared to healthy controls. Sutterella species were found in none of the controls, but in twelve of twenty-three ASD patients. Sutterella species are normally scarce in a healthy microbiota. Nevertheless, in these ASD patients they were the third to eighth highest occurring species [91]. The microbial composition was also studied in duodenal biopsies comparing nineteen autistic children with twenty-one healthy controls [92]. The study did not find major differences at the phylum level; however, at genus und species level some alterations were observed. The genus Burkholderia was significantly increased in autistic participants compared to controls. The genera Actinomyces, Oscillospira, Peptostreptococcus and Ralstonia were also elevated. Moreover, the genus Neisseria was significantly under-represented in autistic children compared to controls. The genera Bacteroides, Devosia, Prevotella and Streptococcus were also decreased. Lower abundances of two species from the genus Bacteroides and the specie Escherichia coli were observed [92]. Compared to other studies, Kushak et al. could not find major differences at phylum level or had similar results as other mentioned studies $[7,35,39,58,89]$. This could be due to the fact, that all the other studies investigated microbial composition from stool samples and not biopsies from duodenal mucosa. The stool samples represented, by and large, microbial composition of the large intestine [93]. The results from Kushak et al. represented microbial composition of the small intestine. The discovery of these minor changes between the groups could also be a result of variation of the microbial composition along the whole GI tract. Kushak's results suggested that microbial changes in the large intestine could have a greater impact on the pathology of ASD than the changes in the small intestine. Therefore, a better representation of the microbial gut milieu could be achieved by collecting mucosal samples from all over the GI-tract for comparison of the microbiota.

Biopsies from the ileum and the caecum of ASD children showed a lower abundance of Bacteroides leading to a significantly higher Firmicutes to Bacteroidetes ratio. Levels of Clostridiales (Firmicutes) were slightly elevated in ASD group, especially the families Lachnospiraceae and Ruminococcaceae and the genus Faecalibacterium. The class Betaproteobacteria (Proteobacteria) was significantly higher abundant in faecal samples of the caecum in ASD patients, within the class Betaproteobacteria the family Alcaligenaceae was the highest abundant. With increasing levels of Bacteroidetes the mRNA levels of SGLT1 in ileum and caecum significantly increased, whereas the mRNA levels of sucrase isomaltase (disaccharidase) decreased in the caecum. With increasing mRNA levels of sucrose isomaltase in the caecum there were significant decreased amounts of Firmicutes [39]. These associations may be of therapeutic interest, as they demonstrated how bacterial composition influenced expressions of the host's sugar transporters and enzymes to consequently alter their nutrients' availability. Therefore, directing the bioactive therapeutic molecules to the small intestine or the colon may be a practical approach to circumvent systematic absorption achieving specifically the modulation of the microbiota in these organs.

A Slovakian study enrolling twenty-nine participants including autistic children, their siblings and healthy children found that the amount of Lactobacillus species was significantly increased (Figure 3) in the faeces of autistic children compared to siblings and healthy controls. Amount of Desulfovibrio and Clostridia species were also increased, whereas Bifidobacterium species were decreased in autistic children (Figure 3) [35]. The authors could associate the severity of behavioural symptoms in autism with the amount of Desulfovibrio species present. Therefore, Desulfovibrio species were considered pathogenic microbes. Interestingly non-autistic siblings of autistic children showed lower abundance of Bacteroidetes and higher abundance of Firmicutes compared to healthy controls with no family history of autism [35]. These findings point to an altered microbial composition in non-autistic as well as autistic siblings compared to healthy controls. Furthermore, non-autistic siblings had lower abundance of Clostridia and Desulfovibrio species and significantly lower counts of Bifidobacterium species compared to autistic children. Therefore, levels of bacterial species could be the tipping factor between autistic or healthy phenotypes, but the small sample size of the study demands to handle the information with care [35]. 
In an Italian study the phyla Fusobacteria and Verrucomicrobia were also represented in lower concentrations (Figure 3) in the faeces of ten autistic children compared to ten healthy controls [7]. Significantly elevated bacteria in autistic children were Akkermansia muciniphila, Anerofilum, Barnesiella intestinihominis, Clostridium species, Dorea species, the family Enterobacteriaceae except for Escherichia coli, which was significantly decreased, Faecalibacterium (especially Faecalibacterium prausnitzii), Roseburia species, Parasutterella excrementihominis, Prevotella copri and Prevotella oris, Turicibacter species [7]. Other higher represented species were Aeromonas, Odirobacter splanchnicus, Parabacteroides species, Porphyromonas, Pseudomonas, and Turicibacter sanguinis. Significantly decreased bacteria in autistic children were Bifidobacterium, Fusobacterium, Oscillospira, Sporobacter, Streptococcus and Subdoligranulum. Lower represented genera in autistic children were Collinsella species except Collinsella aerofaciens, Enterococcus species, Lactobacillus, Lactococcus and Staphylococcus [7].

Strati et al. found significantly elevated abundances of the bacterial genera Collinsella, Corynebacterium, Dorea and Lactobacillus in forty autistic children when compared to healthy children. Lowered levels of Alistipes, Bilophila, Dialister, Parabacteroides, and Veillonella were also found in autistic children. Additionally, the authors found the yeast Candida to be present at increased rates in autistic children. Constipation could be associated with higher levels of Escherichia, Shigella and Clostridium cluster XVIII and lower levels of Gemmiger and Ruminococcus [89]. Lactobacillus species stimulate the immune system to produce IL-22. IL-17 and IL-22 together inhibited the overgrowth of Candida species, however in autistic population the altered diversity of microbial community favoured the growth of Candida species. Furthermore, Candida species, once established in the gut, prevented recolonisation of commensal microbes [89]. Kantarcioglu et al. investigated the abundance of the yeast species Candida in the gut of autistic children, which was elevated. Reportedly this yeast is associated with some autistic behaviour and $60 \%$ of the healthy population is estimated to be asymptomatic carrier of Candida species [94]. Normally, Candida cannot grow in the healthy gut due to the competition with other microbes for space and nutrients and suppression through commensal bacteria. On the other hand, in a dysbiotic environment as frequently observed in the autistic population the yeast proliferates and produces ammonia and toxins. Candida also causes reduction in absorption of minerals and carbohydrates by the host. The release of toxins and ammonia was reported to increase autistic behaviour [94]. Several studies found notably higher abundance of Candida species, especially Candida albicans in the faecal samples of autistic children compared to healthy counterparts $[8,94]$. Overgrowth of the yeast Candida albicans in autistic children can be treated with Nystatin or Fluconazole [94].

Higher occurrence of Clostridium species in the gut was associated with disease severity using the Childhood Autism Rating score (CARs score) [8]. Significantly higher counts of the bacterium Clostridium perfringens were found in faecal samples of autistic children compared to controls. Especially the species Clostridium perfringens producing the beta2-toxin gene was significantly elevated in autistic children. The authors also found a significant relationship between the abundance of the beta2-toxin gene and occurrence of autism [90]. A review suggested that a sub-acute tetanus infection again with a Clostridium species might be the cause of some cases of autism. Clostridium tetani infection only occurs in dysbiotic GI tract, as it is an opportunistic pathogen. It may be inactive for several months until favourable conditions allow its growth. Clostridium tetani produces the neurotoxin tetanus neurotoxin, which crosses the intestinal barrier. Tetanus neurotoxin is then transported via the vagal nerve to the nucleus solitarius and subsequently to the whole CNS. Tetanus neurotoxin inhibits the release of synaptic vesicles containing neurotransmitters by irreversibly cleaving synaptobrevin. Synaptobrevin is a membrane-associated protein, which is important for vesicle stability. Synapses with cleaved synaptobrevin degenerate and lower synaptic activity correlates with diminished social behaviour found in autism (figure 4). Tetanus neurotoxin targets inhibitory neurons releasing GABA or glycine. Purkinje cells in the cerebellum release GABA as neurotransmitter, making them vulnerable to the toxin. Additionally, Purkinje and granular cells express receptors for the neurotoxin. This is in line with the observation of decreased Purkinje and granular cells in autopsies of autistic children [27]. 


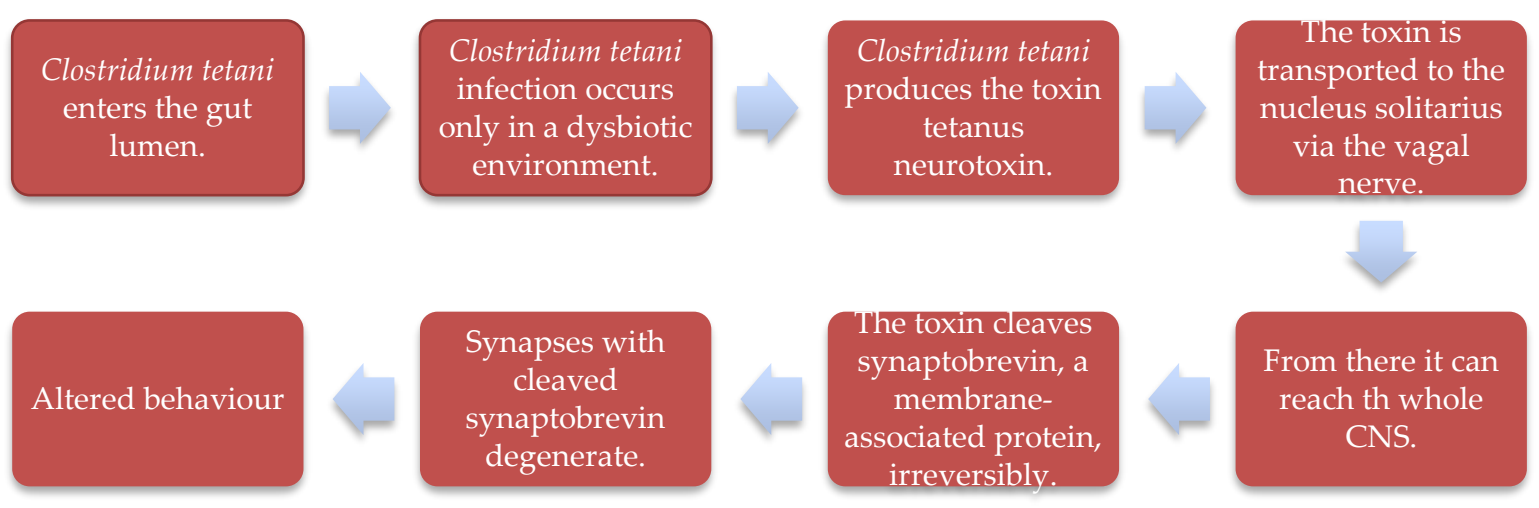

Figure 4. Process of Clostridium tetani infection in the gut. Occurrence of the bacterium Clostridium tetani in the GI-tract of autistic population could lead to altered behaviour.

A simulation by Weston et al. showed the interdependency between the anti-inflammatory genera Bifidobacterium and the pro-inflammatory Clostridia and Desulfovibrio. Bifidobacterium is inhibited by lysozyme and the growth of Desulfovibrio. To some extent, Desulfovibrio thrives on metabolites produced by Bifidobacterium. Growth of Clostridia is inhibited by lysozymes and by a higher abundance of Bifidobacterium. The authors claimed that the growth of Clostridia in the gut with low abundances of Bifidobacterium is a key risk for the development of autism [95]. Another simulated study analysed the microbiome coding for enzymes involved in the metabolism of glutamate and found that these enzymes were underrepresented in autistic microbiome compared to healthy ones. Glutamate is a constituent of the important peptide glutathione, which is an antioxidant and therefore reduces oxidative stress in the cell. This amino acid is also an excitatory neurotransmitter. An imbalance in the CNS between excitation and inhibition has been postulated to contribute to autism [96]. A general observed trend is that Bifidobacterium species are scarcer represented in the guts of autistic children, whereas Clostridia species are higher abundant [97].

Detecting altered microbial composition is especially important to understand how microbial metabolites can modulate gut and neuronal functions. Enterochromaffin cells in the gut produce neurotransmitters in response to luminal composition. Enhanced excitability was observed in colonic neurons induced by the species Lactobacillus reuteri. Intestinal neurons may also be activated through Bacteroides fragilis and Lactobacillus rhamnosus. Sensory neurons in the gut could be activated by the strain Staphylococcus aureus, which could lead to heightened pain perception [98]. All these changes observed in the microbial composition could either be a cause leading to autism or a consequence resulting from the pathology [87]. Microbial compositional changes could occur due to a selfrestricted eating pattern, which is often observed in ASD patients [99]. Apparently, $90 \%$ of autistic children prefer starches, snacks and processed food to non-processed food such as fruits and vegetables. Depleting the gut from nutrients favours some bacteria over others. Microbial composition can react to changed nutrients availability based on its high plasticity [99]. More monoand disaccharides entering the large intestine and thus, changing the microbial composition is a more plausible explanation than just picky eating habits because there is proof of disturbed carbohydrate processing and absorption [39]. Future studies are needed to establish a possible causation. Nevertheless, a significantly changed microbial composition was found in the GI-tracts of autistic population pointing to a correlation between the microbiota and the occurrence of autism. However, some of the aforementioned results stand in contrast to one another. Therefore, conclusive evidence is yet to be established by future studies.

\subsection{Altered concentrations of metabolites and their functional consequences}

\subsubsection{Urinary analysis}


Yap et al. compared urinary metabolite profiles of thirty-nine autistic children, twenty-eight non-autistic siblings and thirty-four healthy controls using NMR spectroscopy. Autistic children were found to excrete significantly more $\mathrm{N}$-methyl-2-pyridone-5-carboxamide, $\mathrm{N}$-methyl nicotinic acid, and $\mathrm{N}$-methyl nicotinamide in their urine. Knowing that nicotinamide derives from tryptophan, this finding suggested autism-associated alterations of the nicotinamide and the tryptophan metabolism [100]. Other significantly elevated metabolites in the urinary profile of ASD children were acetate, dimethylamine, $\mathrm{N}$-acetyl glycoprotein fragments, succinate and taurine. Significantly lower levels of glutamate, hippurate and phenylacetylglutamine were present in the urinary profile of ASD children. Precursors of phenylacetylglutamine and hippurate are phenylacetic acid and benzoic acid, which are both produced by bacterial metabolism [100]. Hippurate is benzoic acid conjugated with glycine [101]. N-methyl-2-pyridone-5-carboxamide, N-methyl nicotinic acid, and Nmethyl nicotinamide are end products of the nicotinamide pathway. It is worth mentioning that comparing healthy siblings to autistic children or neurotypical controls, there were no major differences in the metabolic profile. This indicated that siblings of autistic children exhibited a microbial composition in between their autistic siblings and normal controls resulting in similar metabolites levels [100].

In their urinary metabolite profile of thirty autistic children Gevi et al. found major differences in the tryptophan and purine metabolism compared to thirty neurotypical children. Other significantly perturbed pathways were the vitamin B6 pathway and the phenylalanine- tyrosinetryptophan biosynthesis, among others [102]. Additionally increased levels of tryptophan degradation products were found in the urine suggesting increased production of tryptophan or increased abnormal degradation [99]. Four pathways utilize tryptophan. Indican or indolyl-lactate is produced by bacterial degradation. Through the human metabolism in the kynurenine pathway, kynurenic acid, xanthurenic acid or quinolinic acid are generated. Serotonin and melatonin are produced in another pathway from tryptophan (figure 5). In the autistic metabolome kynurenic acid and melatonin are found in much lower concentration and xanthurenic acid and quinolinic acid are elevated compared to controls. The enzyme kynureninase uses vitamin B6 as cofactor. Therefore, B6 deficiency explains lower concentrations of kynurenic acid and elevated levels of xanthurenic acid and quinolinic acid in the kynurenine pathway [102]. Also, greater amounts of indolyl-3-acetic acid and indolyl-lactate were found in the urine [102] and indole and 3-methylindole in the faeces of autistic children [7]. Following species produce indoles: Escherichia coli, Proteus vulgaris, Paracolobactrum coliform, Achromobacter liguefaciens, Bacteroides species [102] and Clostridium species [7]. Indole is absorbed in the gut whereafter, it is oxidized and sulphated in the liver to indoxyl sulphate. Indoxyl sulphate may lead to an accumulation of several neurotransmitters in the brain by blocking the efflux transporter in the BBB [102]. A study revealed increased excretion of sulphate, sulphite and thiosulphate into the urine. They reasoned that this finding was due to dysfunctional sulphate transporters in the renal tube cells, hindering the reabsorption of these metabolites [100].

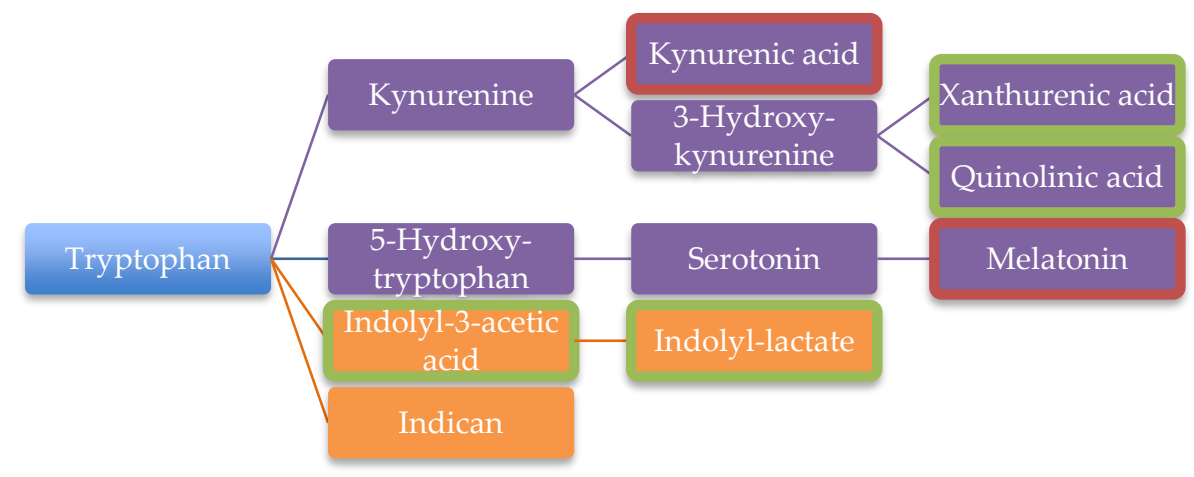

Figure 5. Metabolic pathways of tryptophan. The purple pathways show human metabolism of tryptophan and the orange pathways are observed in bacterial degradation. Green surrounded boxes show elevated metabolites and red surrounded boxes show decreased metabolites in the autistic metabolome [102]. 
A study by Xiong et al. analysed urinary samples from sixty-two autistic and sixty-two neurotypical controls. They found significantly higher concentrations of 3-(3-hydroxyphenyl)- 3hydroxypropionic acid, 3-hydroxyphenylacetic acid and 3-hydroxyhippuric acid in samples of autistic children (figure 6) [103]. These data are in line with the proposition that 3-(3-hydroxyphenyl)3-hydroxypropionic acid induce autistic symptoms by decreasing catecholamine levels in the brain [34]. After oral administration of Vancomycin and supplementation of the probiotic Bifidobacterium to these children the mentioned metabolites significantly decreased [103]. Vancomycin is an antibiotic, which is poorly absorbed by the GI mucosa and has a great effect on gram-positive bacteria such as Clostridia species. The decrease of 3-(3-hydroxyphenyl)-3-hydroxypropionic acid, 3hydroxyphenylacetic acid and 3-hydroxyhippuric acid after treatment suggests that these metabolites are mostly produced by Clostridia species and other overgrown populations in an altered phenylalanine metabolism. The treatment showed improved eye contact and less constipation in autistic children [103]. This finding was in line with the observation that children with altered phenylalanine metabolism, such as phenylketonuria, also often develop autism [104].

Species from Clostridiaceae are known to produce many metabolites such as phenols, p-cresol and indoles, all of which may act as toxins on the human metabolism [7]. P-cresol, which was investigated as a possible biomarker for autism, was elevated in the urinary [102,105] and faecal [7] samples of autistic children (figure 6). P-cresol has an inhibitory effect on the enzyme dopamine-bhydroxylase and thereby regulates the metabolism of the important neurotransmitter dopamine in the brain [104]. As $p$-cresol is only produced in the GI tract, its occurrence in the system correlated with increased permeability of the gut. In addition, $p$-cresol was shown to correlate with autistic behaviour and the severity of the disease [106].

Other metabolites found in altered concentrations were TNF- $\alpha$, free amino acids and quorum sensing molecules (figure 6). TNF- $\alpha$ was found in heightened levels in the faeces of autistic children, which correlated with the severity of GI symptoms [35]. Because increased TNF- $\alpha$ and IL-6 can also be a marker of inflammation in the brain [1], these data re-confirmed a higher level of inflammation in the brains of autistic children. Elevated circulating free amino acid levels were elevated in patients with autism. Glutamate especially was found in higher quantities in the faeces of autistic patients. Glutamate is a neurotransmitter and the excess of it and can lead to apoptosis of neurons [7]. Quorom sensing molecules are signal molecules exchanged between bacteria. PhrCACET1 is a quorum sensing molecule, which can be synthesized by Clostridium acetobutylicum, Clostridium botulinum, Clostridium perfringens, Clostridium difficile and Clostridium tetani. Because PhrCACET1 readily crosses the BBB and Clostridium species are highly abundant in autistic individuals, the effect on PhrCACET1 should be closer investigated [107].

Accumulatively, these data showed several altered metabolites in the urinary profile of autistic children, some even with correlation to autistic behaviour such as $p$-cresol. The metabolic pathways of tryptophan, purine, vitamin B6 and phenylalanine seem perturbed. The most intriguingly changed metabolites in the urine of autistic patients were 3-(3-hydroxyphenyl)-3-hydroxypropionic acid, 3hydroxyphenylacetic acid and 3-hydroxyhippuric acid due to treatment with the antibiotic Vancomycin and the probiotic Bifidobacterium, both of which in combination lead to improvement of autistic behaviour. These metabolites indicated an altered phenylalanine metabolism in autism. A connection was made between decreased levels of these metabolites, changed gut microbial composition through treatment and improved autistic behaviour. We therefore suggest that 3-(3hydroxyphenyl)-3-hydroxypropionic acid, 3-hydroxyphenylacetic acid and 3-hydroxyhippuric acid should be investigated as possible biomarkers for autism. 


\section{Elevated urinary metabolites in ASD patients}

- N-methyl-2-pyridone-5-carboxamide

- N-methyl nicotinic acid

- N-methyl nicotinic acid

- N-acetyl glycoprotein

- Succinate

- Taurine

-Dimethylamine

-3-(3-hydroxyphenyl)-3-hydroxypropionic acid

-3-hydroxyphenylacetic acid

-3-hydroxyhippuric acid

- Tryptophan degradation products

-Xanthurenic acid

-Quinolinic acid

-Indolyl-3-acetic

-Indolyl-lactate

-P-cresol

-TNF- $\alpha$

- Sulphate, sulphite, thiosulphate

\section{Decreased urinary metabolites in ASD patients}

- Glutamate

- Hippurate

- Phenylacetylglutamine

- Kynurenic acid

-Melatonin

Figure 6. Elevated and decreased metabolites in the urinary profiles of ASD children. Significant results are written in bold.

\subsubsection{Blood analysis}

Elevated serotonin levels in the whole blood was observed in $25 \%$ of autistic children (figure 7), thus, a study by Marler et al. aimed to identify a link between hyperserotoninemia and constipation due to reduced motility in the GI tract [108]. Resultantly, co-occurring GI symptoms were observed in children with ASD and children with hyperserotoninemia. However, the relationship between hyperserotoninemia and constipation was not significant in the study [108]. Serotonin serves as a major neurotransmitter in the CNS and the gut. It controls in the GI tract the motility, pain perception and GI secretion. In the brain serotonin is responsible for regulating mood and cognition. In GF mice, higher levels of serotonin were found in the hippocampus and higher levels of tryptophan, the biosynthetic precursor of serotonin, in the blood [56]. Known producers of serotonin are species from Candida, Streptococcus, Escherichia and Enterococcus [55]. Another neurotransmitter found in elevated concentration in autistic children was the inhibitory neurotransmitter GABA [97]. Some Lactobacillus and Bifidobacterium species are known producers of GABA [22,55,109]. Producers of another neurotransmitter, noradrenaline, are species from Bacillus, Enterococcus, Escherichia, Saccharomyces 
and Streptococcus [22,55]. Neurotransmitter produced in the gut acts upon gut epithelial cells to produce active molecules that can be secreted into the periphery or on afferent neurons leading to the CNS.

Autistic children are also known to have significantly lower plasma concentrations of glutathione, homocysteine, methionine and S-adenosylmethione (figure 7) [100]. Sadenosylmethione acts as an important methyldonor in the body and homocysteine is a precursor of s-adenosylmethionine, suggesting perturbations in the sulphur metabolism. Methylation and glutathione are used to lower oxidative stress in the cell. The methylation of nicotinic acid to Nmethyl-2-pyridone-5-carboxamide, $\mathrm{N}$-methyl nicotinic acid, and $\mathrm{N}$-methyl nicotinamide, which were found in increased levels in the urinary profile, consumes even more methyl-donor and so increasing sensitivity to oxidative stress in cells of autistic children [100]. The excretion of heavy metals is difficult in ASD patients due to the fact that glutathione is less available and transmethylation/transsulphuration pathways are altered [70]. Acetaminophen (Paracetamol) is maltolerated by autistic individuals. Mal-toleration occurs due to insufficient capability to sulphate and thus detoxify the drug. This is in total agreement with the fact that people with high 4-cresol blood levels are also known not to tolerate Paracetamol and high levels of 4-cresol are also found in autistic children, as it is produced by some Clostridia species [100].

Examining metabolites in serum and in red blood cells in eleven Canadian autistic children revealed significantly lower concentrations of docosahexaenoic acid, eicosapentaenoic acid, arachidonic acid and lower ratio of omega- 3 to omega- 6 fatty acids in red blood cells compared to fifteen controls (figure 7). There were also significantly lower concentrations of docosahexaenoic acid, arachidonic acid and linoleic acid found in the serum [110]. Decreased fatty acids levels in patients might be a direct result of the eating pattern observed in autistic individuals and not a cause leading to autism. As constituents of neuronal membranes, docosahexaenoic acid and arachidonic acid both play a role in the maturing of neuronal networks. Docosahexaenoic acid is also important for the myelination of axons [111]. Both fatty acids are also modulator of gene expression in the cell, which could also be a contributing epigenetic factor for autism [110].

Another metabolite with altered concentration in autistic patients was thiamine-pyrophosphate (TPP). The concentration in the plasma was significantly decreased compared to controls. Furthermore, precursor (thiamine and thiamine-monophosphate) concentrations were similar in both groups (figure 7) [112]. Lower concentration of TPP could result from an impaired excretion from producing cells into the bloodstream or an impaired uptake from the gut lumen. Indeed, over $40 \%$ of the gut microbiota are known to produce TPP. TPP is a cofactor to many enzymes in the mitochondria, most importantly transketolase in the oxidative pentose-phosphate pathway [112]. A decreased function of transketolase leads to an accumulation of the substrate for the oxidative pathway, which in turn inhibits the reductive pathway of pentose-phosphate-pathway resulting in lower concentration of NADPH. Lower abundance of NADPH leads to an increased oxidative stress in the cell [112].

Increased amounts of lipopolysaccharides were also found in the blood of autistic individuals (figure 7). Lipopolysaccharides are produced by bacteria and may cause inflammation in the brain due to pro-inflammatory nature and the ability of activating microglia. Levels of heightened lipopolysaccharides in autistic individuals correlated with elevated IL-6 levels, which also indicated an inflammation. An inflammation compromises the integrity of the BBB, facilitating the accumulation of heavy metals in the cerebellum, thus worsening the symptoms [15,42].

The above data indicates an alteration in the sulphur metabolism and a reduced reduction capability in the cells of autistic people. Decreased levels s-adenosylmethionine and its precursor homocysteine lead to decreased methylation processes. Due to reduced methylation processes and decreased levels of glutathione found in autistic individuals the reduction capacity is significantly minimized. Decreased levels of TPP lead to a lessened reduction capacity, as they are a cofactor of transketolase inhibiting the production NADPH. 


\section{Elevated metabolites in the blood of ASD patients}

- Serotonin (Candida, Streptococcus, Escherichia and Enterococcus)

- GABA (Lactobacillus and Bifidobacterium species)

-4-cresol (Clostridia species)

-Lipopolysaccharides

Decreased metabolites in the blood of ASD patients
-Methionine
-S-adenosylmethionine
-Homocysteine
- Glutathione
-Docosahexaenoic acid
-Eicosapentaenoic acid
-Arachidonic acid
-Thiamine-pyrophosphate

Figure 7. Summary of elevated and decreased metabolites in the blood of autistic patients.

Significant results are written in bold.

\subsubsection{Short chain fatty acids}

SCFAs, mainly butyrate, propionate and acetate [113], are produced as fermentation products by gut bacteria. In autistic children lower concentrations of the total amount of SCFAs are found $[7,97]$, providing the evidence for a reduced fermentation capacity of the microbiota. Nevertheless, propionate and acetate are found in higher levels in autistic children suggesting that butyrate production is dramatically reduced [7]. SCFAs cross the BBB and influence early brain development by modulating production of the neurotransmitter serotonin and dopamine [7]. These acids bind to two free fatty acid receptors (FFA2 and FFA3) found in several areas of the brain, which are G-protein coupled receptors $[17,55,79]$. The reabsorption of water and electrolytes in the colon is also to some degree regulated by SCFAs [7]. They also have modulatory functions on T-cells and cytokine production [45]. Positively correlated with total amount of SCFA were Faecalibacterium, Ruminococcus and Bifidobacterium genera, while the amount of Bacteroides species was correlated with propionic acid in autistic individuals [7]. Bacteroides species, Porphyromonadaceae and Parabacteroides were positively correlated with the amount of propionate and butyrate in autistic individuals. Alcaligenaceae is also positively correlated with propionate, whereas the Bacteroidaceae is negatively correlated with acetate and total amount of SCFA in autistic children [72].

Intraventricular injection of propionate into rat pups caused behavioural and physiological changes as such observed in autism [97]. An accumulation of propionic acid can lead to prolonged neurodevelopment and seizures [23]. Clostridia species are known to produce exotoxins and propionate and thus create an inflammatory status, which might worsen autistic symptoms [89]. An oxidative milieu in the cell, as seen in autistic individuals, leads among others to malfunctioning of mitochondrial enzymes and production of reactive nitrogen species. The reactive nitrogen species interact with propionic acid and produce 3-nitropropionic acid. This acid acts as a mitochondrial neurotoxin, which irreversibly inhibits the function of the enzyme succinate dehydrogenase, the producer of one NADH. Propionic acid is also converted to propionyl-CoA and enters the citric acid cycle at the enzyme succinyl-CoA-dehydrogenase. At this entry two enzymes, which produce NADH are surpassed. NADH is a substrate for the electron transport chain and consequently for ATP production. This explains the lower activity of ETC enzyme complex I due to reduced substrate concentration [114]. Propionate is involved in up-regulating the cyclic-adenosin-monophosphate response element binding protein (CREB). CREB regulates synaptic plasticity, memory formation and the reward system [115]. A study by Frye et al. compared mitochondrial function of lymphoblastoid cells from autistic subjects and controls after incubating them in propionic acid and 
introduction to reactive oxygen species (ROS). Cells from autistic individuals consumed propionic acid much faster as a fuel, suggesting a compensatory measurement for energy production. Propionic acid metabolised by healthy lymphoblastoid cells exerted beneficial effects on the cells such as increased respiration. Autistic cells had a stronger reaction to ROS after propionic acid incubation than non-autistic lymphoblastoid cells. The consequence was, among others, an increased proton leakage from the mitochondria inter-membrane space and hence a reduced respiratory capacity [114].

Butyrate is used as an energy source in colonocytes [45]. It is involved in regulating inflammatory and oxidative state of the mucosal cells, visceral sensitivity and motility and reinforces the mucosal barrier in the intestines. Butyrate modulates the biosynthesis of catecholamines and neurotransmitters of the CNS and autonomic nervous system [99]. It prevents carcinogenesis and inflammation in the gut via its anti-inflammatory properties [113]. Butyrate is said to be the most important SCFA in humans as it exhibits neuroprotective features. It promotes memory formation and neuronal plasticity through epigenetic modulation [76]. Histones are protein complexes storing the DNA in a compact form. In butyrate-rich environment histones remain acetylated. Acetylated histones prevent DNA from transcription or replication, as the sites for transcription and replication are not accessible in the compact DNA [116]. Thus, butyrate is implicated in epigenetic regulation of gene expression in human cells $[76,117,118]$. In some neurodegenerative models butyrate was found to ameliorate symptoms [76]. There were also decreased levels of butyrate found in patients with Crohn's disease [113]. Even if not conclusive, the above data suggest an involvement of increased acetate and propionate in the pathophysiology of autism.

\subsection{Other etiologies for autism}

Over one hundred genes are associated with the pathology of autism and up to four hundred genes are implicated in elevation of the susceptibility to autism [34]. In some genes of autistic individuals single mutations were found. These affected genes accounted for brain development in utero as well as during infancy [28]. Most of the differing gene expression lead to an altered structure of the CNS or the ENS, the enteric nervous system [34]. Autism has been hypothesised to be linked to abnormalities of the neural crest development during pregnancy [119]. Inoue et al. found in peripheral blood mononuclear cells of autistic children, 1056 up-regulated and 517 down-regulated genes, respectively [120]. Comorbidities as Fragile X syndrome, tuberous sclerosis or Rett disorder have also been observed in ASD patients (figure 1) [28]. Six Japanese autistic children were compared to six healthy controls regarding microbial composition by collecting faeces and blood. The genus Faecalibacterium was higher and Blautia lower represented in the faeces of autistic children. Moreover, there were several up- and down regulated genes in autistic children compared to healthy ones. Interestingly some genes, which are involved in IFN-gamma mediated pathway correlated with the higher and lower abundance of Faecalibacterium and Blautia. These data are in accord with the finding that IFN-gamma is also elevated in autistic children, which suggests a chronic inflammation in these children. Faecalibacterium prausnitzii is also found in higher abundance in Crohn's disease, which has been linked to autism [31] and obese patients [120].

It was observed that children having allergic reactions in the first year of life showed several behavioural deficits later in life and less social skills as observed in autistic population compared to controls [85]. Another aetiology for autism in mice was bisphenol A exposure in utero. Bisphenol A changed the methylation and thus, the expression of the gene for BDNF [121]. BDNF is important for survival of neurons, growth and differentiation of newly formed neurons as well as new formations of synapses [55]. Altered BDNF expression could also lead to obesity and diabetes mellitus type 2 . Intriguingly, treatment with resveratrol, a food derived polyphenol, could prevent autism induced by bisphenol A in animal models demonstrating the potential of diet in treating ASD [121].

The data above indicates that several genes, which are involved in CNS development and inflammation pathways are altered in autistic individuals providing several targets for a better understanding of the mechanisms involved in the disease and possible therapeutic targets.

\section{Possible therapeutic measures acting on the microbiota}




\subsection{Prebiotics}

Dietary prebiotics were defined as "a selectively fermented ingredient that results in specific changes in the composition and/or activity of the gastrointestinal microbiota, thus conferring benefit(s) upon host health" [59,122].

Grimaldi et al. investigated the role of the prebiotic galactooligosaccharide in a synthetic colon model comparing diluted faecal samples from autistic children and control regarding changes in bacterial composition and metabolic profile. The administration of the prebiotic led to significant increases in Bifidobacteria species in both autistic and control models [97]. Slightly significant increases of the Clostridium cluster XI and decreases of Veillonellaeceae were observed only in the ASD group. However, there were also smaller decreases in Sutterella species, Bacteroides, Clostridial cluster IX, Escherichia coli, Veillonellaceae, Ruminococcus species, Clostridium leptum and Faecalibacterium prausnitzii after administration of the prebiotic in autistic children. The combined prebiotic containing fructo- and galactooligosaccharides in the same ratio as contained in human breast milk is also known to stimulating growth of Bifidobacteria [55]. Bifidobacteria are considered as beneficial bacteria [12]. In rodents this combination of prebiotics also increased the expression of neurotransmitters in the hippocampus [55]. Galactooligosaccharide also affected SCFA production, increasing butyrate and decreasing propionate, so the SCFA produced by bacteria were mainly acetate and butyrate. Overproduction of GABA, a dysfunction of the neurotransmitter system in the brain of ASD could also be normalised by the prebiotic treatment [97].

Prebiotics are not widely researched as a treatment option for autistic individuals. Nonetheless, existing data point to the fact that prebiotic treatment can cause microbial compositional alterations in autistic children that could result in amelioration of autistic behaviour. As mentioned before, methodologies to deliver therapeutics to the colon could be envisaged to directly reach the microbiota without the interference of the digestive system.

\subsection{Probiotics}

Probiotics contain living microorganisms and are administered to promote health by stimulating immunity, strengthening the intestinal barrier, increasing the expression of mucine, reducing the overgrowth of pathogens and producing vitamins and antioxidants [1]. Most common used probiotics are species from Bifidobacterium and Lactobacillus [42].

The administration of the probiotic cocktail "Children Dophilus" three times a day for four months shifted the Bacteroidetes/Firmicutes ratio towards more Bacteroidetes and less Firmicutes. The abundances of the genera Desulfovibrio and Bifidobacterium also increased in autistic children. The cocktail contained strains from Lactobacillus, Bifidobacterium and Streptococcus [35]. The administration of the probiotic mixture of Lactobacillus rhamnosus, Bifidobacterium infantis, Bifidobacterium longus, Lactobacillus helveticus, Lactobacillus reuteri and Lactobacillus paracasei to autistic children improved comorbid GI problems [99]. Lactobacillus reuteri on its own could reverse intestinal inflammation caused by lipopolysaccharides [24]. Furthermore, the bacterium Lactobacillus reuteri influenced the posterior pituitary gland to produce more oxytocin. Oxytocin is a hypothalamic hormone positively influencing social behaviour [22]. It remains to be determined whether increased levels of oxytocin in autistic children could ameliorate their behaviour in social interactions.

The administration of Bifidobacterium species to mice for 8 weeks resulted in higher concentration of arachidonic and docosahexaenoic acid in their brains [79]. This altered fatty acids composition in the brain exerted a beneficial effect on neurogenesis, neurotransmission and preventing oxidative stress. Learning and memory were positively affected by these fatty acids [79]. The administration of Lactobacillus rhamnosus in mice on the other hand reduced corticosterone levels and thereby positively influenced anxiety and depression. Another observed effect was altered levels of GABA and its receptors throughout the brain. These effects could only be achieved with an intact vagal nerve, which reconfirms the role of the vagal nerve as a major route of communication between the brain and the gut microbiota [79].

Hsiao et al. treated gut permeability and altered microbial composition by oral administration of the commensal microbe Bacteroides fragilis in MIA mice showing that even autistic features were 
improved. Interestingly the probiotic also corrected altered expression of the tight junction proteins CLDN-8 and CLDN-15 in the colon. Heightened levels of IL-6 found in the colon of MIA mice were normalized, which leads to improved gut barrier function. A metabolite, which was found in significantly higher levels in MIA mice than in controls, is 4-ethylphenylsulfate, which is similar to p-cresol and is produced by Clostridia species. P-cresol is investigated as a biomarker for autism in humans. The administration of 4-ethylphenylsulfate to mice surprisingly created anxiety like behaviour, which reaffirms that $\mathrm{p}$-cresol has a similar effect on humans. Bacteroides fragilis also corrected serum levels of 4-ethylphenylsulfate in MIA mice [16].

Probiotics are known to alter the microbial composition in the gut of autistic children. Behavioural changes observed in MIA mice after administration of Bacteroides fragilis raise the interesting question whether the same result also applies to humans [123].

Probiotic used

Children Dophilus

(Bifidobacterium, Lactobacillus,

Streptococcus species)

Lactobacillus rhamnosus,

Bifidobacterium infantis,

Bifidobacterium longus,

Lactobacillus helveticus,

Lactobacillus reuteri and

Lactobacillus paracasei

Lactobacillus reuteri

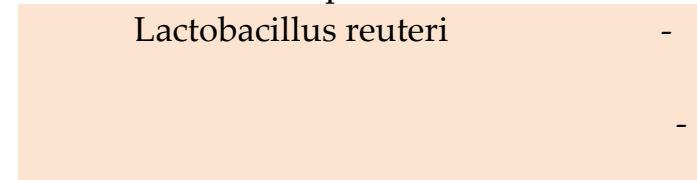

Bifidobacterium species

Lactobacillus rhamnosus

Bacteroides fragilis
Effects

Amelioration of Firmicutes to

Bacteroidetes ratio

Improvement of GI problems

Humans [99]

\section{Studies}

Humans [35]

$$
\begin{aligned}
& \text { Reversing inflammation caused } \\
& \text { through lipopolysaccharides } \\
& \text { Stimulating the production of } \\
& \text { Oxytocin }
\end{aligned}
$$

Elevation of arachidonic and

docosahexaenoic acid concentration in

the brain

- Reduction of corticosterone levels

- Alterations of GABA levels

- Improvement of autistic features

- Correction of tight junction

proteins expression in the colon
Mice [79]

Rats [24]

Mice [79]

Mice [16]

Table 2. Non-exhaustive list of human studies supplementing probiotics in autistic individuals.

\subsection{Faecal Microbiota transplantation}

In contrast to probiotic treatment, where just some bacterial strains are supplemented, faecal microbiota transplantation (FMT) ensures the transfer of several hundred bacterial strains. A healthy individual donates a stool sample [60]. The donated stool sample is normally used freshly within eight hours, or within eight weeks when directly frozen after the passage [124]. FMT are potentially problematic, as donors could transfer opportunistic pathogens or infections to recipients. A thorough screening of donors before donation minimises the risk but in the early stages of infections patients could be asymptomatic and unknowingly transfer infections [125]. Despite these uncertainties, FMT is an established and efficient therapy in recurrent Clostridium difficile infection [59,60].

Kang et al. studied the impact on the microbial composition and the course of GI and autistic symptoms in eighteen autistic children after "Microbiota Transfer Therapy" [60]. The transfer followed over a course of 7-8 weeks after oral administration of Vancomycin and bowel cleansing. The results were impressing, because there was a decrease of $80 \%$ of the GI symptoms such as constipation, diarrhoea or abdominal pain. The improvement lasted at least for eight weeks but there 
was no follow-up after that time frame. Not just gastrointestinal but also autistic symptoms improved after faecal microbiota transfer [60]. The main consequence of faecal transplantation was an increased bacterial diversity and altered abundances of some bacteria such as the genera Bifidobacterium, Prevotella and Desulfovibrio. This suggests that GI and behavioural symptoms in autistic children trace back to lesser microbial diversity probably through modulating metabolites [60].

Therefore, FMT is an exciting new treatment option, which could be considered for ASD patients with dysbiosis.

\subsection{Other treatment strategies}

Amelioration of behavioural symptoms could also be reached through oral treatment with Vancomycin, which is an antibiotic specific to bacteria such as Clostridiaceae [7]. This finding suggests a connection between Clostridiaceae and autistic behaviour. However, the amelioration of the symptoms did not last longer than the treatment duration. This was attributed to spore forming capacity of these bacteria. Spores of bacteria can survive the antibiotic treatment and proliferate afterwards [7]. Also, restricted gluten- and/or casein-free diet seemed to positively affect the social and cognitive problems in children with autism [7]. The theory is that these food components are metabolised to opioid-like substances and act in the CNS $[99,126]$. However, the evidence is rather weak, as opioid-like substances were not found in autistic individuals. This restricted diet should only be considered if there are allergic reactions to the abovementioned food components [70,99].

Interestingly, higher rates of autism have been reported in people with dark skin and in regions with limited solar irradiation. Significantly lower levels of 25-hydroxy-Vitamin D in serum were found in autistic children in comparison to controls. In a study vitamin D supplementation was given to rat pups to treat autism induced by propionic acid. The authors discovered that vitamin $\mathrm{D}$ has neuroprotective features and less DNA breaks were detected in the brains of rats treated with vitamin $\mathrm{D}$ compared to untreated propionic acid induced rats. These results, however, could only be obtained, when the pups were treated with vitamin D before inducing autism. Instead, the study suggested that vitamin D exhibits rather a preventive effect rather than a therapeutic one [127]. The administration of vitamin B1 to autistic children was also suggested to ameliorate symptoms due to vitamin B1 producing bacteria Prevotella being found in lower abundances in autistic children [15].

One publication even suggested the introduction of helminths, which are parasitic worms, into the gut ecosystem to achieve a greater diversity. One argument was that before daily hygienic measures were introduced in our society, helminths were a normal part of the gut microbiota and the prevalence for autism was smaller [119]. This hypothesis was experimentally supported by the data that immune activated rats showed less anxiety in dangerous situations, which is atypical for animals. In addition, these immune-activated rats when exposed to helminths, showed normal behaviour [119]. Obviously, these treatment options were poorly examined and are not recommended as a treatment option for ASD patients before thorough investigations.

\section{Discussion and Conclusion}

It remains yet to be unequivocally determined whether dysbiosis is a factor causing autism or if the disease is causing the microbial alterations. The mutual correlation, however, has been undoubtedly confirmed by many animal and human studies between ASD and alterations in the microbiota [120]. To narrow down the mechanisms of disease further studies with bigger sample sizes are required, since the works summarized in this review showed varying degrees of statistical significance on top of small sample sizes. Our review also showed big variations of methodologies and outcome measures calling for standardisation of the treatment regimens to achieve higher statistical significance and a better comparison between the study results.

This review article demonstrated significant changes in microbial composition of autistic population. The discovery and understanding the connection between the microbiota and autism is especially important to find new treatment options and a possible cure for this disease. Treatment of autistic behaviour based on microbial compositional alterations such as probiotics and faecal microbiota transfer showed promising results in GF-mice and autistic individuals $[16,24,35,60,79,99]$. 
Especially, faecal microbiota transfer ameliorated autistic behaviour and GI symptoms significantly in autistic children [60]. For treatment with probiotics it is crucial to determine the optimal dose as a wrong dosage of probiotics bear the intrinsic risk of inducing sepsis as a side effect [123]. For widespread use of these treatments more studies must be conducted to finetune treatment, mode of delivery, its duration and doses.

Often observed changes in ASD children were a generally decreased bacterial diversity compared to control and the significantly decreased Bacteroidetes to Firmicutes ratio $[35,39,58,89]$. Elevated abundances of Clostridium species in autistic individuals are correlated with the severity of autistic behaviour [8]. An evolutionary reason why the microbiota could alter our behaviour is that the more social the host is, the more the microbes can spread through the society [128]. We suspect that most of these microbial composition alterations occur due to reduced mRNA expressions of disaccharidases and hexose transporters in the gut epithelium of ASD patients [39]. More mono- and disaccharides entering the colon favours some bacterial species over others and consequently leads to an altered metabolome in the gut affecting the host.

Significantly lower plasma concentration of S-adenosylmethionine, glutathione [100] and thiamine-pyrophosphate [112] shows perturbation in transmethylation processes in the body and diminished reduction capability of the cell. A new possible family of biomarkers for autism is suggested in our review including 3-(3-hydroxyphenyl)-3-hydroxypropionic acid, 3hydroxyphenylacetic acid and 3-hydroxyhippuric acid. These metabolites were increased in the urinary profile of ASD patients linking ASD to an abnormal phenylalanine metabolism. As a consequence of treating ASD patients with an antibiotic and a probiotic, these suggested biomarkers decreased in the urine of autistic patients. Additionally, autistic behaviour was ameliorated in the patients due to this treatment [34]. Our suggested biomarkers need to be validated, but there is a major possibility that these metabolites are part of an autistic-behaviour-causing metabolome.

In conclusion the strong correlation between the microbiota and the brain suggests further investigations into the microbiota-gut-brain-axis for an in-depth study of mechanisms leading to the pathology of autism.

Author Contributions: “Conceptualization, P.S. and M.H.M.; Methodology, P.S.; Validation, M.H.M.; Formal Analysis, P.S.; Investigation, P.S.; Resources, P.S.; Data Curation, P.S.; Writing-Original Draft Preparation, P.S.; Writing-Review \& Editing, P.S. and M.H.M.; Visualization, P.S.; Supervision, M.H.M.; Project Administration, M.H.M. and P.S.."

Funding: This research received no external funding.

Acknowledgments: We would like to thank Joshua Dan Itsch Csucker, Nilujana Srikantha and David Wolfer for useful comments and the review of this paper.

Conflicts of Interest: The authors declare no conflict of interest.

$\begin{array}{ll}\text { Abbreviations } \\ \text { ASD } & \text { Autism Spectrum disorder } \\ \text { BBB } & \text { Blood-brain-barrier } \\ \text { BDNF } & \text { Brain derived neurotropic factor } \\ \text { CLDN } & \text { Claudine } \\ \text { DNA } & \text { Deoxyribonucleic acid } \\ \text { ETC } & \text { Electron transport chain } \\ \text { FMT } & \text { Faecal Microbiota transplantation } \\ \text { GABA } & \text { Gamma-amino-butyric acid } \\ \text { GF } & \text { Germ-free } \\ \text { GI } & \text { Gastrointestinal } \\ \text { GLT2 } & \text { Glucose transporter 2 } \\ \text { MIA } & \text { Maternal immune activation } \\ \text { NADH } & \text { Nicotineamide-adenine-dinucleotide } \\ \text { NADPH } & \text { Nicotineamide-adenine-dinukleotide-phosphate } \\ \text { poly(I:C) } & \text { Polyinosinic:polycytidylic acid }\end{array}$


ROS Reactive oxygen species

SCFAs Short chain fatty acids

SGLT Sodium dependent glucose transporter 1

TPP Thiamine-pyrophosphate

\section{References}

1. Santocchi, E.; Guiducci, L.; Fulceri, F.; Billeci, L.; Buzzigoli, E.; Apicella, F.; Calderoni, S.; Grossi, E.; Morales, M.A.; Muratori, F. Gut to brain interaction in Autism Spectrum Disorders: a randomized controlled trial on the role of probiotics on clinical, biochemical and neurophysiological parameters. BMC Psychiatry 2016, 16, 183, doi:10.1186/s12888-016-0887-5.

2. Bik, E.M. The Hoops, Hopes, and Hypes of Human Microbiome Research. Yale J Biol Med 2016, 89, 363373.

3. Sharon, G.; Sampson, T.R.; Geschwind, D.H.; Mazmanian, S.K. The Central Nervous System and the Gut Microbiome. Cell 2016, 167, 915-932, doi:10.1016/j.cell.2016.10.027.

4. Buie, T. Potential Etiologic Factors of Microbiome Disruption in Autism. Clin Ther 2015, 37, 976-983, doi:10.1016/j.clinthera.2015.04.001.

5. Redinbo, M.R. The microbiota, chemical symbiosis, and human disease. J Mol Biol 2014, 426, 3877-3891, doi:10.1016/j.jmb.2014.09.011.

6. Viggiano, D.; Ianiro, G.; Vanella, G.; Bibbo, S.; Bruno, G.; Simeone, G.; Mele, G. Gut barrier in health and disease: focus on childhood. Eur Rev Med Pharmacol Sci 2015, 19, 1077-1085.

7. De Angelis, M.; Piccolo, M.; Vannini, L.; Siragusa, S.; De Giacomo, A.; Serrazzanetti, D.I.; Cristofori, F.; Guerzoni, M.E.; Gobbetti, M.; Francavilla, R. Fecal microbiota and metabolome of children with autism and pervasive developmental disorder not otherwise specified. PLoS One 2013, 8, e76993, doi:10.1371/journal.pone.0076993.

8. Iovene, M.R.; Bombace, F.; Maresca, R.; Sapone, A.; Iardino, P.; Picardi, A.; Marotta, R.; Schiraldi, C.; Siniscalco, D.; Serra, N., et al. Intestinal Dysbiosis and Yeast Isolation in Stool of Subjects with Autism Spectrum Disorders. Mycopathologia 2017, 182, 349-363, doi:10.1007/s11046-016-0068-6.

9. Mulle, J.G.; Sharp, W.G.; Cubells, J.F. The gut microbiome: a new frontier in autism research. Curr Psychiatry Rep 2013, 15, 337, doi:10.1007/s11920-012-0337-0.

10. Rosenfeld, C.S. Microbiome Disturbances and Autism Spectrum Disorders. Drug Metab Dispos 2015, 43, 1557-1571, doi:10.1124/dmd.115.063826.

11. Mangiola, F.; Ianiro, G.; Franceschi, F.; Fagiuoli, S.; Gasbarrini, G.; Gasbarrini, A. Gut microbiota in autism and mood disorders. World J Gastroenterol 2016, 22, 361-368, doi:10.3748/wjg.v22.i1.361.

12. Arora, S.K.; Dewan, P.; Gupta, P. Microbiome: Paediatricians' perspective. Indian J Med Res 2015, 142, 515-524, doi:10.4103/0971-5916.171275.

13. Marler, S.; Ferguson, B.J.; Lee, E.B.; Peters, B.; Williams, K.C.; McDonnell, E.; Macklin, E.A.; Levitt, P.; Margolis, K.G.; Beversdorf, D.Q., et al. Association of Rigid-Compulsive Behavior with Functional Constipation in Autism Spectrum Disorder. J Autism Dev Disord 2017, 47, 1673-1681, doi:10.1007/s10803017-3084-6.

14. Mezzelani, A.; Raggi, M.E.; Marabotti, A.; Milanesi, L. Ochratoxin A as possible factor trigging autism and its male prevalence via epigenetic mechanism. Nutr Neurosci 2016, 19, 43-46, doi:10.1179/1476830515z.000000000186. 
15. Kang, D.W.; Park, J.G.; Ilhan, Z.E.; Wallstrom, G.; Labaer, J.; Adams, J.B.; Krajmalnik-Brown, R. Reduced incidence of Prevotella and other fermenters in intestinal microflora of autistic children. PLoS One 2013, 8, e68322, doi:10.1371/journal.pone.0068322.

16. Hsiao, E.Y.; McBride, S.W.; Hsien, S.; Sharon, G.; Hyde, E.R.; McCue, T.; Codelli, J.A.; Chow, J.; Reisman, S.E.; Petrosino, J.F., et al. Microbiota modulate behavioral and physiological abnormalities associated with neurodevelopmental disorders. Cell 2013, 155, 1451-1463, doi:10.1016/j.cell.2013.11.024.

17. Dinan, T.G.; Cryan, J.F. The Microbiome-Gut-Brain Axis in Health and Disease. Gastroenterol Clin North Am 2017, 46, 77-89, doi:10.1016/j.gtc.2016.09.007.

18. Muszer, M.; Noszczynska, M.; Kasperkiewicz, K.; Skurnik, M. Human Microbiome: When a Friend Becomes an Enemy. Arch Immunol Ther Exp (Warsz) 2015, 63, 287-298, doi:10.1007/s00005-015-0332-3.

19. Petra, A.I.; Panagiotidou, S.; Hatziagelaki, E.; Stewart, J.M.; Conti, P.; Theoharides, T.C. Gut-MicrobiotaBrain Axis and Its Effect on Neuropsychiatric Disorders With Suspected Immune Dysregulation. Clin Ther 2015, 37, 984-995, doi:10.1016/j.clinthera.2015.04.002.

20. Quigley, E.M. Basic Definitions and Concepts: Organization of the Gut Microbiome. Gastroenterol Clin North Am 2017, 46, 1-8, doi:10.1016/j.gtc.2016.09.002.

21. Rinaldi, A. Piecing together a different picture: A host of new studies on autism have begun decoding the longstanding puzzle of its causes. EMBO Rep 2016, 17, 1690-1695, doi:10.15252/embr.201643502.

22. Dinan, T.G.; Cryan, J.F. Gut instincts: microbiota as a key regulator of brain development, ageing and neurodegeneration. J Physiol 2017, 595, 489-503, doi:10.1113/jp273106.

23. Berding, K.; Donovan, S.M. Microbiome and nutrition in autism spectrum disorder: current knowledge and research needs. Nutr Rev 2016, 74, 723-736, doi:10.1093/nutrit/nuw048.

24. Navarro, F.; Liu, Y.; Rhoads, J.M. Can probiotics benefit children with autism spectrum disorders? World J Gastroenterol 2016, 22, 10093-10102, doi:10.3748/wjg.v22.i46.10093.

25. Manchia, M.; Fanos, V. Targeting aggression in severe mental illness: The predictive role of genetic, epigenetic, and metabolomic markers. Prog Neuropsychopharmacol Biol Psychiatry 2017, 77, 32-41, doi:10.1016/j.pnpbp.2017.03.024.

26. Mayer, E.A.; Padua, D.; Tillisch, K. Altered brain-gut axis in autism: comorbidity or causative mechanisms? Bioessays 2014, 36, 933-939, doi:10.1002/bies.201400075.

27. Bolte, E.R. Autism and Clostridium tetani. Med Hypotheses 1998, 51, 133-144.

28. Madore, C.; Leyrolle, Q.; Lacabanne, C.; Benmamar-Badel, A.; Joffre, C.; Nadjar, A.; Laye, S. Neuroinflammation in Autism: Plausible Role of Maternal Inflammation, Dietary Omega 3, and Microbiota. Neural Plast 2016, 2016, 3597209, doi:10.1155/2016/3597209.

29. Diaz Heijtz, R. Fetal, neonatal, and infant microbiome: Perturbations and subsequent effects on brain development and behavior. Semin Fetal Neonatal Med 2016, 21, 410-417, doi:10.1016/j.siny.2016.04.012.

30. Fulceri, F.; Morelli, M.; Santocchi, E.; Cena, H.; Del Bianco, T.; Narzisi, A.; Calderoni, S.; Muratori, F. Gastrointestinal symptoms and behavioral problems in preschoolers with Autism Spectrum Disorder. Dig Liver Dis 2016, 48, 248-254, doi:10.1016/j.dld.2015.11.026.

31. Reddy, B.L.; Saier, M.H. Autism and our intestinal microbiota. In J Mol Microbiol Biotechnol, (c) 2015 S. Karger AG, Basel.: Switzerland, 2015; Vol. 25, pp. 51-55.

32. Wakefield, A.J. The gut-brain axis in childhood developmental disorders. J Pediatr Gastroenterol Nutr 2002, 34 Suppl 1, S14-17.

33. Yu, L.; Wu, Y.; Wu, B.L. Genetic architecture, epigenetic influence and environment exposure in the pathogenesis of Autism. Sci China Life Sci 2015, 58, 958-967, doi:10.1007/s11427-015-4941-1. 
34. Li, Q.; Zhou, J.M. The microbiota-gut-brain axis and its potential therapeutic role in autism spectrum disorder. Neuroscience 2016, 324, 131-139, doi:10.1016/j.neuroscience.2016.03.013.

35. Tomova, A.; Husarova, V.; Lakatosova, S.; Bakos, J.; Vlkova, B.; Babinska, K.; Ostatnikova, D. Gastrointestinal microbiota in children with autism in Slovakia. Physiol Behav 2015, 138, 179-187, doi:10.1016/j.physbeh.2014.10.033.

36. Ding, H.T.; Taur, Y.; Walkup, J.T. Gut Microbiota and Autism: Key Concepts and Findings. J Autism Dev Disord 2017, 47, 480-489, doi:10.1007/s10803-016-2960-9.

37. Hornig, M. The role of microbes and autoimmunity in the pathogenesis of neuropsychiatric illness. Curr Opin Rheumatol 2013, 25, 488-795, doi:10.1097/BOR.0b013e32836208de.

38. Naviaux, R.K. Metabolic features of the cell danger response. Mitochondrion 2014, 16, 7-17, doi:10.1016/j.mito.2013.08.006.

39. Williams, B.L.; Hornig, M.; Buie, T.; Bauman, M.L.; Cho Paik, M.; Wick, I.; Bennett, A.; Jabado, O.; Hirschberg, D.L.; Lipkin, W.I. Impaired carbohydrate digestion and transport and mucosal dysbiosis in the intestines of children with autism and gastrointestinal disturbances. PLoS One 2011, 6, e24585, doi:10.1371/journal.pone.0024585.

40. van De Sande, M.M.; van Buul, V.J.; Brouns, F.J. Autism and nutrition: the role of the gut-brain axis. Nutr Res Rev 2014, 27, 199-214, doi:10.1017/s0954422414000110.

41. Fiorentino, M.; Sapone, A.; Senger, S.; Camhi, S.S.; Kadzielski, S.M.; Buie, T.M.; Kelly, D.L.; Cascella, N.; Fasano, A. Blood-brain barrier and intestinal epithelial barrier alterations in autism spectrum disorders. Mol Autism 2016, 7, 49, doi:10.1186/s13229-016-0110-z.

42. Fond, G.; Boukouaci, W.; Chevalier, G.; Regnault, A.; Eberl, G.; Hamdani, N.; Dickerson, F.; Macgregor, A.; Boyer, L.; Dargel, A., et al. The "psychomicrobiotic": Targeting microbiota in major psychiatric disorders: A systematic review. Pathol Biol (Paris) 2015, 63, 35-42, doi:10.1016/j.patbio.2014.10.003.

43. Vuong, H.E.; Hsiao, E.Y. Emerging Roles for the Gut Microbiome in Autism Spectrum Disorder. Biol Psychiatry 2017, 81, 411-423, doi:10.1016/j.biopsych.2016.08.024.

44. Wakefield, A.J.; Puleston, J.M.; Montgomery, S.M.; Anthony, A.; O'Leary, J.J.; Murch, S.H. Review article: the concept of entero-colonic encephalopathy, autism and opioid receptor ligands. Aliment Pharmacol Ther 2002, 16, 663-674.

45. Rose, S.; Bennuri, S.C.; Murray, K.F.; Buie, T.; Winter, H.; Frye, R.E. Mitochondrial dysfunction in the gastrointestinal mucosa of children with autism: A blinded case-control study. PLoS One 2017, 12, e0186377, doi:10.1371/journal.pone.0186377.

46. Gondalia, S.V.; Palombo, E.A.; Knowles, S.R.; Cox, S.B.; Meyer, D.; Austin, D.W. Molecular characterisation of gastrointestinal microbiota of children with autism (with and without gastrointestinal dysfunction) and their neurotypical siblings. Autism Res 2012, 5, 419-427, doi:10.1002/aur.1253.

47. Ianiro, G.; Bibbo, S.; Gasbarrini, A.; Cammarota, G. Therapeutic modulation of gut microbiota: current clinical applications and future perspectives. Curr Drug Targets 2014, 15, 762-770.

48. Douglas-Escobar, M.; Elliott, E.; Neu, J. Effect of intestinal microbial ecology on the developing brain. JAMA Pediatr 2013, 167, 374-379, doi:10.1001/jamapediatrics.2013.497.

49. Rescigno, M. Intestinal microbiota and its effects on the immune system. Cell Microbiol 2014, 16, 10041013, doi:10.1111/cmi.12301.

50. Rook, G.A.; Raison, C.L.; Lowry, C.A. Microbiota, immunoregulatory old friends and psychiatric disorders. Adv Exp Med Biol 2014, 817, 319-356, doi:10.1007/978-1-4939-0897-4_15. 
51. Groer, M.W.; Gregory, K.E.; Louis-Jacques, A.; Thibeau, S.; Walker, W.A. The very low birth weight infant microbiome and childhood health. Birth Defects Res C Embryo Today 2015, 105, 252-264, doi:10.1002/bdrc.21115.

52. SM, O.M.; Stilling, R.M.; Dinan, T.G.; Cryan, J.F. The microbiome and childhood diseases: Focus on brain-gut axis. Birth Defects Res C Embryo Today 2015, 105, 296-313, doi:10.1002/bdrc.21118.

53. Principi, N.; Esposito, S. Gut microbiota and central nervous system development. J Infect 2016, 73, 536546, doi:10.1016/j.jinf.2016.09.010.

54. Borre, Y.E.; O'Keeffe, G.W.; Clarke, G.; Stanton, C.; Dinan, T.G.; Cryan, J.F. Microbiota and neurodevelopmental windows: implications for brain disorders. Trends Mol Med 2014, 20, 509-518, doi:10.1016/j.molmed.2014.05.002.

55. Dinan, T.G.; Stilling, R.M.; Stanton, C.; Cryan, J.F. Collective unconscious: how gut microbes shape human behavior. J Psychiatr Res 2015, 63, 1-9, doi:10.1016/j.jpsychires.2015.02.021.

56. Borre, Y.E.; Moloney, R.D.; Clarke, G.; Dinan, T.G.; Cryan, J.F. The impact of microbiota on brain and behavior: mechanisms \& therapeutic potential. Adv Exp Med Biol 2014, 817, 373-403, doi:10.1007/978-14939-0897-4_17.

57. Rook, G.A.; Lowry, C.A.; Raison, C.L. Hygiene and other early childhood influences on the subsequent function of the immune system. Brain Res 2015, 1617, 47-62, doi:10.1016/j.brainres.2014.04.004.

58. Dinan, T.G.; Cryan, J.F. The impact of gut microbiota on brain and behaviour: implications for psychiatry. Curr Opin Clin Nutr Metab Care 2015, 18, 552-558, doi:10.1097/mco.0000000000000221.

59. Mohajeri, M.H.; Brummer, R.J.M.; Rastall, R.A.; Weersma, R.K.; Harmsen, H.J.M.; Faas, M.; Eggersdorfer, M. The role of the microbiome for human health: from basic science to clinical applications. Eur J Nutr 2018, doi:10.1007/s00394-018-1703-4.

60. Kang, D.W.; Adams, J.B.; Gregory, A.C.; Borody, T.; Chittick, L.; Fasano, A.; Khoruts, A.; Geis, E.; Maldonado, J.; McDonough-Means, S., et al. Microbiota Transfer Therapy alters gut ecosystem and improves gastrointestinal and autism symptoms: an open-label study. Microbiome 2017, 5, 10, doi:10.1186/s40168-016-0225-7.

61. Nicolas, C.; Benitez, P.R.; Riano, M.O.; Canencia, L.M.; Mercurio, C.; Fernandez, M.S.; Luna, M.S.; Jorge, A.T. Preeclampsia: Long-term effects on pediatric disability. J Neonatal Perinatal Med 2016, 9, 41-48, doi:10.3233/npm-16915065.

62. Curran, E.A.; Dalman, C.; Kearney, P.M.; Kenny, L.C.; Cryan, J.F.; Dinan, T.G.; Khashan, A.S. Association Between Obstetric Mode of Delivery and Autism Spectrum Disorder: A Population-Based Sibling Design Study. JAMA Psychiatry 2015, 72, 935-942, doi:10.1001/jamapsychiatry.2015.0846.

63. Curran, E.A.; Cryan, J.F.; Kenny, L.C.; Dinan, T.G.; Kearney, P.M.; Khashan, A.S. Obstetrical Mode of Delivery and Childhood Behavior and Psychological Development in a British Cohort. J Autism Dev Disord 2016, 46, 603-614, doi:10.1007/s10803-015-2616-1.

64. Son, J.S.; Zheng, L.J.; Rowehl, L.M.; Tian, X.; Zhang, Y.; Zhu, W.; Litcher-Kelly, L.; Gadow, K.D.; Gathungu, G.; Robertson, C.E., et al. Comparison of Fecal Microbiota in Children with Autism Spectrum Disorders and Neurotypical Siblings in the Simons Simplex Collection. PLoS One 2015, 10, e0137725, doi:10.1371/journal.pone.0137725.

65. Louis, P. Does the human gut microbiota contribute to the etiology of autism spectrum disorders? Dig Dis Sci 2012, 57, 1987-1989, doi:10.1007/s10620-012-2286-1.

66. Knight, R.; Callewaert, C.; Marotz, C.; Hyde, E.R.; Debelius, J.W.; McDonald, D.; Sogin, M.L. The Microbiome and Human Biology. Annu Rev Genomics Hum Genet 2017, 18, 65-86, doi:10.1146/annurevgenom-083115-022438. 
67. Gonzalez, A.; Stombaugh, J.; Lozupone, C.; Turnbaugh, P.J.; Gordon, J.I.; Knight, R. The mind-bodymicrobial continuum. Dialogues Clin Neurosci 2011, 13, 55-62.

68. Labouesse, M.A.; Langhans, W.; Meyer, U. Long-term pathological consequences of prenatal infection: beyond brain disorders. Am J Physiol Regul Integr Comp Physiol 2015, 309, R1-r12, doi:10.1152/ajpregu.00087.2015.

69. Ibi, D.; Yamada, K. Therapeutic Targets for Neurodevelopmental Disorders Emerging from Animal Models with Perinatal Immune Activation. Int $J$ Mol Sci 2015, 16, 28218-28229, doi:10.3390/ijms161226092.

70. Lazaro, C.P.; Ponde, M.P.; Rodrigues, L.E. Opioid peptides and gastrointestinal symptoms in autism spectrum disorders. Rev Bras Psiquiatr 2016, 38, 243-246, doi:10.1590/1516-4446-2015-1777.

71. Alam, R.; Abdolmaleky, H.M.; Zhou, J.R. Microbiome, inflammation, epigenetic alterations, and mental diseases. Am J Med Genet B Neuropsychiatr Genet 2017, 174, 651-660, doi:10.1002/ajmg.b.32567.

72. Borghi, E.; Borgo, F.; Severgnini, M.; Savini, M.N.; Casiraghi, M.C.; Vignoli, A. Rett Syndrome: A Focus on Gut Microbiota. Int J Mol Sci 2017, 18, doi:10.3390/ijms18020344.

73. Latalova, K.; Hajda, M.; Prasko, J. Can gut microbes play a role in mental disorders and their treatment? Psychiatr Danub 2017, 29, 28-30.

74. Sherwin, E.; Sandhu, K.V.; Dinan, T.G.; Cryan, J.F. May the Force Be With You: The Light and Dark Sides of the Microbiota-Gut-Brain Axis in Neuropsychiatry. CNS Drugs 2016, 30, 1019-1041, doi:10.1007/s40263-016-0370-3.

75. Skosnik, P.D.; Cortes-Briones, J.A. Targeting the ecology within: The role of the gut-brain axis and human microbiota in drug addiction. Med Hypotheses 2016, 93, 77-80, doi:10.1016/j.mehy.2016.05.021.

76. Mohajeri, M.H.; La Fata, G.; Steinert, R.E.; Weber, P. Relationship between the gut microbiome and brain function. Nutr Rev 2018, 76, 481-496, doi:10.1093/nutrit/nuy009.

77. Stilling, R.M.; Dinan, T.G.; Cryan, J.F. Microbial genes, brain \& behaviour - epigenetic regulation of the gut-brain axis. Genes Brain Behav 2014, 13, 69-86, doi:10.1111/gbb.12109.

78. Rao, M.; Gershon, M.D. The bowel and beyond: the enteric nervous system in neurological disorders. Nat Rev Gastroenterol Hepatol 2016, 13, 517-528, doi:10.1038/nrgastro.2016.107.

79. Liu, X.; Cao, S.; Zhang, X. Modulation of Gut Microbiota-Brain Axis by Probiotics, Prebiotics, and Diet. J Agric Food Chem 2015, 63, 7885-7895, doi:10.1021/acs.jafc.5b02404.

80. Martin, C.R.; Mayer, E.A. Gut-Brain Axis and Behavior. Nestle Nutr Inst Workshop Ser 2017, 88, 45-53, doi:10.1159/000461732.

81. Fung, T.C.; Olson, C.A.; Hsiao, E.Y. Interactions between the microbiota, immune and nervous systems in health and disease. Nat Neurosci 2017, 20, 145-155, doi:10.1038/nn.4476.

82. Zhang, Y.J.; Li, S.; Gan, R.Y.; Zhou, T.; Xu, D.P.; Li, H.B. Impacts of gut bacteria on human health and diseases. Int J Mol Sci 2015, 16, 7493-7519, doi:10.3390/ijms16047493.

83. Nguyen, T.L.; Vieira-Silva, S.; Liston, A.; Raes, J. How informative is the mouse for human gut microbiota research? Dis Model Mech 2015, 8, 1-16, doi:10.1242/dmm.017400.

84. Schumann, C.M.; Amaral, D.G. Stereological analysis of amygdala neuron number in autism. J Neurosci 2006, 26, 7674-7679, doi:10.1523/JNEUROSCI.1285-06.2006.

85. de Theije, C.G.; Bavelaar, B.M.; Lopes da Silva, S.; Korte, S.M.; Olivier, B.; Garssen, J.; Kraneveld, A.D. Food allergy and food-based therapies in neurodevelopmental disorders. Pediatr Allergy Immunol 2014, 25, 218-226, doi:10.1111/pai.12149.

86. Vuong, H.E.; Yano, J.M.; Fung, T.C.; Hsiao, E.Y. The Microbiome and Host Behavior. Annu Rev Neurosci 2017, 40, 21-49, doi:10.1146/annurev-neuro-072116-031347. 
87. Mayer, E.A.; Tillisch, K.; Gupta, A. Gut/brain axis and the microbiota. J Clin Invest 2015, 125, 926-938, doi:10.1172/jci76304.

88. Lichtman, J.S.; Sonnenburg, J.L.; Elias, J.E. Monitoring host responses to the gut microbiota. Isme j 2015, 9, 1908-1915, doi:10.1038/ismej.2015.93.

89. Strati, F.; Cavalieri, D.; Albanese, D.; De Felice, C.; Donati, C.; Hayek, J.; Jousson, O.; Leoncini, S.; Renzi, D.; Calabro, A., et al. New evidences on the altered gut microbiota in autism spectrum disorders. Microbiome 2017, 5, 24, doi:10.1186/s40168-017-0242-1.

90. Finegold, S.M.; Summanen, P.H.; Downes, J.; Corbett, K.; Komoriya, T. Detection of Clostridium perfringens toxin genes in the gut microbiota of autistic children. Anaerobe 2017, 45, 133-137, doi:10.1016/j.anaerobe.2017.02.008.

91. Williams, B.L.; Hornig, M.; Parekh, T.; Lipkin, W.I. Application of novel PCR-based methods for detection, quantitation, and phylogenetic characterization of Sutterella species in intestinal biopsy samples from children with autism and gastrointestinal disturbances. MBio 2012, 3, doi:10.1128/mBio.00261-11.

92. Kushak, R.I.; Winter, H.S.; Buie, T.M.; Cox, S.B.; Phillips, C.D.; Ward, N.L. Analysis of the Duodenal Microbiome in Autistic Individuals: Association With Carbohydrate Digestion. J Pediatr Gastroenterol Nutr 2017, 64, e110-e116, doi:10.1097/mpg.0000000000001458.

93. Mayer, E.A.; Knight, R.; Mazmanian, S.K.; Cryan, J.F.; Tillisch, K. Gut microbes and the brain: paradigm shift in neuroscience. J Neurosci 2014, 34, 15490-15496, doi:10.1523/jneurosci.3299-14.2014.

94. Kantarcioglu, A.S.; Kiraz, N.; Aydin, A. Microbiota-Gut-Brain Axis: Yeast Species Isolated from Stool Samples of Children with Suspected or Diagnosed Autism Spectrum Disorders and In Vitro Susceptibility Against Nystatin and Fluconazole. Mycopathologia 2016, 181, 1-7, doi:10.1007/s11046-0159949-3.

95. Weston, B.; Fogal, B.; Cook, D.; Dhurjati, P. An agent-based modeling framework for evaluating hypotheses on risks for developing autism: effects of the gut microbial environment. Med Hypotheses 2015, 84, 395-401, doi:10.1016/j.mehy.2015.01.027.

96. Heberling, C.; Dhurjati, P. Novel systems modeling methodology in comparative microbial metabolomics: identifying key enzymes and metabolites implicated in autism spectrum disorders. Int J Mol Sci 2015, 16, 8949-8967, doi:10.3390/ijms16048949.

97. Grimaldi, R.; Cela, D.; Swann, J.R.; Vulevic, J.; Gibson, G.R.; Tzortzis, G.; Costabile, A. In vitro fermentation of B-GOS: impact on faecal bacterial populations and metabolic activity in autistic and non-autistic children. FEMS Microbiol Ecol 2017, 93, doi:10.1093/femsec/fiw233.

98. Wang, Y.; Kasper, L.H. The role of microbiome in central nervous system disorders. Brain Behav Immun 2014, 38, 1-12, doi:10.1016/j.bbi.2013.12.015.

99. De Angelis, M.; Francavilla, R.; Piccolo, M.; De Giacomo, A.; Gobbetti, M. Autism spectrum disorders and intestinal microbiota. Gut Microbes 2015, 6, 207-213, doi:10.1080/19490976.2015.1035855.

100. Yap, I.K.; Angley, M.; Veselkov, K.A.; Holmes, E.; Lindon, J.C.; Nicholson, J.K. Urinary metabolic phenotyping differentiates children with autism from their unaffected siblings and age-matched controls. J Proteome Res 2010, 9, 2996-3004, doi:10.1021/pr901188e.

101. Lees, H.J.; Swann, J.R.; Wilson, I.D.; Nicholson, J.K.; Holmes, E. Hippurate: the natural history of a mammalian-microbial cometabolite. J Proteome Res 2013, 12, 1527-1546, doi:10.1021/pr300900b.

102. Gevi, F.; Zolla, L.; Gabriele, S.; Persico, A.M. Urinary metabolomics of young Italian autistic children supports abnormal tryptophan and purine metabolism. Mol Autism 2016, 7, 47, doi:10.1186/s13229-0160109-5. 
103. Xiong, X.; Liu, D.; Wang, Y.; Zeng, T.; Peng, Y. Urinary 3-(3-Hydroxyphenyl)-3-hydroxypropionic Acid, 3-Hydroxyphenylacetic Acid, and 3-Hydroxyhippuric Acid Are Elevated in Children with Autism Spectrum Disorders. Biomed Res Int 2016, 2016, 9485412, doi:10.1155/2016/9485412.

104. Clayton, T.A. Metabolic differences underlying two distinct rat urinary phenotypes, a suggested role for gut microbial metabolism of phenylalanine and a possible connection to autism. FEBS Lett 2012, 586, 956-961, doi:10.1016/j.febslet.2012.01.049.

105. Yang, Y.; Tian, J.; Yang, B. Targeting gut microbiome: A novel and potential therapy for autism. Life Sci 2018, 194, 111-119, doi:10.1016/j.lfs.2017.12.027.

106. Vasquez, A. Biological plausibility of the gut-brain axis in autism. Ann N Y Acad Sci 2017, 1408, 5-6, doi:10.1111/nyas.13516.

107. Wynendaele, E.; Verbeke, F.; Stalmans, S.; Gevaert, B.; Janssens, Y.; Van De Wiele, C.; Peremans, K.; Burvenich, C.; De Spiegeleer, B. Quorum Sensing Peptides Selectively Penetrate the Blood-Brain Barrier. PLoS One 2015, 10, e0142071, doi:10.1371/journal.pone.0142071.

108. Marler, S.; Ferguson, B.J.; Lee, E.B.; Peters, B.; Williams, K.C.; McDonnell, E.; Macklin, E.A.; Levitt, P.; Gillespie, C.H.; Anderson, G.M., et al. Brief Report: Whole Blood Serotonin Levels and Gastrointestinal Symptoms in Autism Spectrum Disorder. J Autism Dev Disord 2016, 46, 1124-1130, doi:10.1007/s10803015-2646-8.

109. Reardon, S. Gut-brain link grabs neuroscientists. In Nature, England, 2014; Vol. 515, pp. 175-177.

110. Jory, J. Abnormal fatty acids in Canadian children with autism. Nutrition 2016, 32, 474-477, doi:10.1016/j.nut.2015.10.019.

111. Weiser, M.J.; Butt, C.M.; Mohajeri, M.H. Docosahexaenoic Acid and Cognition throughout the Lifespan. Nutrients 2016, 8, 99, doi:10.3390/nu8020099.

112. Anwar, A.; Marini, M.; Abruzzo, P.M.; Bolotta, A.; Ghezzo, A.; Visconti, P.; Thornalley, P.J.; Rabbani, N. Quantitation of plasma thiamine, related metabolites and plasma protein oxidative damage markers in children with autism spectrum disorder and healthy controls. Free Radic Res 2016, 50, S85-s90, doi:10.1080/10715762.2016.1239821.

113. Pifer, R.; Sperandio, V. The Interplay between the Microbiota and Enterohemorrhagic Escherichia coli. Microbiol Spectr 2014, 2, doi:10.1128/microbiolspec.EHEC-0015-2013.

114. Frye, R.E.; Rose, S.; Chacko, J.; Wynne, R.; Bennuri, S.C.; Slattery, J.C.; Tippett, M.; Delhey, L.; Melnyk, S.; Kahler, S.G., et al. Modulation of mitochondrial function by the microbiome metabolite propionic acid in autism and control cell lines. Transl Psychiatry 2016, 6, e927, doi:10.1038/tp.2016.189.

115. Slattery, J.; MacFabe, D.F.; Kahler, S.G.; Frye, R.E. Enteric Ecosystem Disruption in Autism Spectrum Disorder: Can the Microbiota and Macrobiota be Restored? Curr Pharm Des 2016, 22, 6107-6121.

116. Lei, E.; Vacy, K.; Boon, W.C. Fatty acids and their therapeutic potential in neurological disorders. Neurochem Int 2016, 95, 75-84, doi:10.1016/j.neuint.2016.02.014.

117. Aw, W.; Fukuda, S. Toward the comprehensive understanding of the gut ecosystem via metabolomicsbased integrated omics approach. Semin Immunopathol 2015, 37, 5-16, doi:10.1007/s00281-014-0456-2.

118. Ghaisas, S.; Maher, J.; Kanthasamy, A. Gut microbiome in health and disease: Linking the microbiomegut-brain axis and environmental factors in the pathogenesis of systemic and neurodegenerative diseases. Pharmacol Ther 2016, 158, 52-62, doi:10.1016/j.pharmthera.2015.11.012.

119. Beales, D.L. Biome depletion in conjunction with evolutionary mismatches could play a role in the etiology of neurofibromatosis 1. Med Hypotheses 2015, 84, 305-314, doi:10.1016/j.mehy.2015.01.014.

120. Inoue, R.; Sakaue, Y.; Sawai, C.; Sawai, T.; Ozeki, M.; Romero-Perez, G.A.; Tsukahara, T. A preliminary investigation on the relationship between gut microbiota and gene expressions in peripheral 
mononuclear cells of infants with autism spectrum disorders. Biosci Biotechnol Biochem 2016, 80, 24502458, doi:10.1080/09168451.2016.1222267.

121. Diaz-Gerevini, G.T.; Repossi, G.; Dain, A.; Tarres, M.C.; Das, U.N.; Eynard, A.R. Beneficial action of resveratrol: How and why? Nutrition 2016, 32, 174-178, doi:10.1016/j.nut.2015.08.017.

122. La Fata, G.; Rastall, R.A.; Lacroix, C.; Harmsen, H.J.M.; Mohajeri, M.H.; Weber, P.; Steinert, R.E. Recent Development of Prebiotic Research-Statement from an Expert Workshop. Nutrients 2017, 9, doi:10.3390/nu9121376.

123. Umbrello, G.; Esposito, S. Microbiota and neurologic diseases: potential effects of probiotics. J Transl Med 2016, 14, 298, doi:10.1186/s12967-016-1058-7.

124. Aroniadis, O.C.; Brandt, L.J. Fecal microbiota transplantation: past, present and future. Curr Opin Gastroenterol 2013, 29, 79-84, doi:10.1097/MOG.0b013e32835a4b3e.

125. Petrof, E.O.; Claud, E.C.; Gloor, G.B.; Allen-Vercoe, E. Microbial ecosystems therapeutics: a new paradigm in medicine? Benef Microbes 2013, 4, 53-65, doi:10.3920/bm2012.0039.

126. Whiteley, P. Nutritional management of (some) autism: a case for gluten- and casein-free diets? Proc Nutr Soc 2015, 74, 202-207, doi:10.1017/s0029665114001475.

127. Alfawaz, H.A.; Bhat, R.S.; Al-Ayadhi, L.; El-Ansary, A.K. Protective and restorative potency of Vitamin $\mathrm{D}$ on persistent biochemical autistic features induced in propionic acid-intoxicated rat pups. BMC Complement Altern Med 2014, 14, 416, doi:10.1186/1472-6882-14-416.

128. Schmidt, C. Mental health: thinking from the gut. Nature 2015, 518, S12-15, doi:10.1038/518S13a. 